\title{
Neuronal ROS-Induced Glial Lipid Droplet Formation is Altered by Loss of Alzheimer's Disease-associated Genes
}

\author{
Matthew J. Moulton ${ }^{1,2}$, Scott Barish ${ }^{1,2}$, Isha Ralhan ${ }^{3,4}$, Jinlan Chang ${ }^{3}$, Lindsey D. \\ Goodman $^{1,2}$, Jake G. Harland ${ }^{1,2}$, Paul C. Marcogliese ${ }^{1,2}$, Jan O. Johansson ${ }^{7}$, Maria \\ S. Ioannou ${ }^{3,4,5,6}$, and Hugo J. Bellen ${ }^{1,2,8,9,10}$
}

${ }^{1}$ Department of Molecular and Human Genetics, Baylor College of Medicine, Houston, TX 77030, USA

2Jan and Dan Duncan Neurological Research Institute, Texas Children's Hospital, Houston, TX 77030, USA

${ }^{3}$ Department of Physiology, University of Alberta, Edmonton, AB, T6G 2R3

${ }^{4}$ Group on Molecular and Cell Biology of Lipids, University of Alberta, Edmonton, AB, T6G 2R3

${ }^{5}$ Department of Cell Biology, University of Alberta, Edmonton, AB, T6G 2R3

${ }^{6}$ Neuroscience and Mental Health Institute, Edmonton, AB, T6G 2R3

${ }^{7}$ Artery Therapeutics, Inc. San Ramon, California, USA

${ }^{8}$ Program in Developmental Biology, Baylor College of Medicine, Houston, TX 77030, USA

${ }^{9}$ Department of Neuroscience, Baylor College of Medicine, Houston, TX 77030, USA

${ }^{10}$ Howard Hughes Medical Institute, Baylor College of Medicine, Houston, TX 77030, USA 


\section{Summary}

A growing list of Alzheimer's disease (AD) genetic risk factors is being identified, but

3 the contribution of these genetic mutations to disease remains largely unknown. Accumulating

4 data support a role of lipid dysregulation and excessive ROS in the etiology of AD. Here, we

5 identified cell-specific roles for eight AD risk-associated genes in ROS-induced glial lipid

6 droplet (LD) formation. We demonstrate that ROS-induced glial LD formation requires two

7 ABCA transporters $(A B C A 1$ and $A B C A 7)$ in neurons, the APOE receptor $(L R P 1)$, endocytic

8 genes (PICALM, CD2AP, and AP2A2) in glia, and retromer genes (VPS26 and VPS35) in both

9 neurons and glia. Moreover, ROS strongly enhances A $\beta 42$-toxicity in flies and A $\beta 42$-plaque

10 formation in mice. Finally, an ABCA1-activating peptide restores glial LD formation in the

11 APOE4-associated loss of LD. This study places AD risk factors in a neuron-to-glia lipid transfer 12 pathway with a critical role in protecting neurons from ROS-induced toxicity.

\section{Keywords}

15 peroxidated lipid transfer, Alzheimer's disease, GWAS, retromer, PICALM, CD2AP, AP2A2,

$16 \quad A B C A 1, A B C A 7$, and $L R P 1$

\section{Introduction}

Alzheimer's disease (AD) is a neurodegenerative disorder characterized by memory loss

20 and cognitive impairment (Rogaeva et al., 2006). AD affects $~ 2 \%$ of the American population and

21 defines $\sim 70 \%$ of dementia cases (Masdeu, 2020). AD is pathologically defined by the aberrant

22 accumulation of Amyloid- $\beta$ peptides $(A \beta)$ into extracellular plaques and hyperphosphorylated-Tau

23 into neurofibrillary tangles (NFT). A $\beta$-plaques primarily consist of A $\beta 42$ fragments formed by the 
24 sequential cleavage of neuronally-expressed amyloid precursor protein (APP) (Behl, 1997; Karch

25 and Goate, 2015; LaFerla and Green, 2012; Rogaeva et al., 2006). Tau, encoded by MAPT, is

26 expressed in neurons, and is required for the assembly and stability of microtubules (MTs). Tau

27 hyperphosphorylation inhibits its normal activity and drives its fibrillization into NFT (Mietelska-

28 Porowska et al., 2014; Wang and Mandelkow, 2016). Both A $\beta$-plaques and NFT are neurotoxic in

29 model organisms and the amyloid cascade hypothesis postulates that A $\beta$-plaque formation drives

30 NFT formation (Bloom, 2014; Götz et al., 2007, 2011; Hardy et al., 2002). Thus, A $\beta$ is a major

31 focus in determining how $\mathrm{AD}$ is initiated and has been a target of many AD therapeutics (Bloom,

32 2014).

AD is currently classified as familial (FAD) or sporadic (SAD). FAD accounts for 2-3\%

34 of AD cases and is associated with autosomal dominant mutations in APP, PSEN1, or PSEN2

35 (Giau et al., 2019; Zhu et al., 2015). It is hypothesized that SAD is caused by a combination of

36 genetic and environmental risk factors (De Strooper and Karran, 2016). In fact, A $\beta$-plaques can

37 form with age or due to trauma in the absence of cognitive impairment, supporting the hypothesis

38 that a combination of genetic and environmental insults are required to induce disease (De Strooper

39 and Karran, 2016). Of interest, multiple genome-wide association studies (GWAS) have been

40 performed on post-mortem AD tissue, identifying over 40 potential genetic risk factors that are

41 significantly associated with disease (Kunkle et al., 2019; Lambert et al., 2013). Interestingly,

42 some of the SNPs identified in these studies are in or near genes that encode proteins involved in

43 lipid regulation (e.g. TREM2, ABCA7) and clathrin-mediated endocytosis (e.g. BIN1, CD2AP,

44 AP2A2, PICALM, and RIN3), but the consequences of disrupting these genes/pathways in specific

45 cell types needs further examination. (Karch and Goate, 2015; Wellington, 2004). The well-

46 established AD-risk allele, the $\varepsilon 4$ allele of Apolipoprotein E (APOE), APOE4, is by far the highest 
47 genetic risk factor in these studies. APOE4 is found in $40-60 \%$ of AD individuals, varying by

48 geographic region, and has a highly significant association with age of AD onset (Karch and Goate,

49 2015; Ward et al., 2012). Homozygous carriers for APOE4 are 8-12 times more likely to develop

50 AD than non-carriers (Michaelson, 2014). In contrast, individuals carrying the $\varepsilon 2$ allele of $A P O E$

51 (APOE2) have reduced risk of developing $\mathrm{AD}$, supporting that it is neuroprotective against $\mathrm{AD}$

52 development (Conejero-Goldberg et al., 2014; Huang and Mahley, 2014; Huynh et al., 2017; Li et

53 al., 2020). While APOE functions to mediate lipid transfer between cells, the APOE4 variant has

54 reduced lipid transport capacity (Hatters et al., 2006; Liu et al., 2017).

55 In addition to genetic risk factors, environmental insults likely modulate severity and onset

56 of disease, including cellular oxidative stress caused by accumulation of reactive oxygen species

57 (ROS). ROS can oxidize and damage proteins, lipids, and nucleic acids (Butterfield and Mattson,

58 2020; Grimm and Eckert, 2017). When properly regulated, ROS can provide beneficial effects to

59 the cell (Ristow and Schmeisser, 2011; Thapa and Carroll, 2017) but damagingly high levels of

60 ROS can occur with age as cellular control mechanisms become depleted, such as decreased

61 antioxidant enzyme expression (Grimm and Eckert, 2017). Recent studies have found evidence of

62 ROS elevation, specifically lipid peroxidation byproducts, in post-mortem tissue from individuals

63 with pre-AD diagnoses, including preclinical AD and mild cognitive impairment as well as in AD

64 brains (Allan Butterfield and Boyd-Kimball, 2018; Berbée et al., 2017; Bradley-Whitman and

65 Lovell, 2015; Bradley et al., 2012; Butterfield, 2020; Peña-Bautista et al., 2019; Zabel et al., 2018).

66 As AD progresses, ROS production is likely exacerbated by A $\beta 42$-mediated neurotoxicity (Allan

67 Butterfield and Boyd-Kimball, 2018; Tönnies and Trushina, 2017) and persistent

68 neuroinflammation (Bisht et al., 2018; Regen et al., 2017), thus creating a vicious cycle that can

69 drive disease progression. 
The complexity of AD pathogenesis and progression is further expanded by the observation that many AD risk genes are expressed in glia in addition to neurons, suggesting that disruptions

72 of these genes may impact multiple cell types in the brain. There is increasing evidence for an

73 important role of dysregulation of glial lipid metabolism in AD (Kunkle et al., 2019; Marioni et

74 al., 2018; Di Paolo and Kim, 2011; Wong et al., 2017). TREM2 and the phospholipase, PLCG2,

75 control lipid metabolism in microglia and may aid the transition of microglia to a diseaseassociated state (Andreone et al., 2020; Nugent et al., 2020). Additionally, lipid sensing by TREM2

77 and lipid transport by ABCA7 is linked to the clearance of A $\beta$ by glial cells (Aikawa et al., 2018;

78 Wang et al., 2015). Glia are also important in the secretion of APOE, which can bind A $\beta$ and

79 facilitate its clearance (Fan et al., 2009; Robert et al., 2017). Interestingly, Alois Alzheimer

80 repeatedly described "adipose saccules" in glial cells of AD patients over a century ago (A.

81 Alzheimer, 1911; Alzheimer, 1907; Stelzmann et al., 1995) but, the link between

82 neurodegeneration and lipid droplet (LD) accumulation in glia wasn't described until recently

83 (Van Den Brink et al., 2018; Liu et al., 2015; Zhang and Liu, 2017). In flies, we showed that

84 elevated levels of ROS induces the formation of glial LDs by transferring peroxidated lipids

85 produced in neurons to glia in a process mediated by the apolipoprotein, Glial Lazarillo (Glaz;

86 homolog to human APOD), whose function can be replaced by human APOE (Liu et al., 2017)

87 (Figure 1). The transfer of lipids from cultured vertebrate neurons that are stressed and physically

88 separated from glia in a dish has also been documented to be dependent on APOE (Ioannou et al.,

89 2019a; Liu et al., 2017). Glial LDs provide some neuroprotective capacity when ROS levels are

90 elevated (Van Den Brink et al., 2018; Liu et al., 2017). In the Drosophila visual system, pigment

91 glia that surround photoreceptor neurons, transport lactate to neurons through monocarboxylate

92 transporters. Lactate is converted to pyruvate and Acetyl-CoA in order to support the TCA cycle 
93 in mitochondria. However, defective mitochondria are unable to optimally utilize this energy

94 source and simultaneously producing ROS, which activates JNK and SREBP transcription factors

95 that drive lipid synthesis using Acetyl-CoA. Newly generated lipids become peroxidated in the

96 presence of ROS and are subsequently transported intracellularly via fatty acid transport proteins

97 (FatP) to a heretofore unknown lipid exporter (Figure 1). After lipid export from neurons, lipids

98 bind to apolipoproteins which allow extracellular lipid transfer and paracrine cellular uptake of

99 lipids. The nearby pigment glia express the apolipoprotein, Glaz, allowing for lipid transfer to glia,

100 via an unknown mechanism, for eventual sequestration of peroxidated lipids in LDs (Figure 1).

101 The production and transfer of lipids from neurons to glia is a highly dose dependent process and

102 reducing many of the players involved in the process by $50 \%$, including Lactate dehydrogenase,

103 Pyruvate dehydrogenase, fatp, and Glaz causes a very significant reduction in the accumulation of

104 LD in pigment glia (Liu et al., 2015, 2017). Moreover, APOE2/3 can restore LD formation in a

105 Glaz loss-of-function mutant background, but APOE4 cannot restore this function, leading to

106 accelerated neurodegeneration (Liu et al., 2017). Human APOE4 has a limited lipid-binding

107 ability, suggesting that lipid clearance may be critical in AD (Hatters et al., 2006). It has been

108 suggested that if the lipid binding affinity of APOE4 could be enhanced, neurodegeneration could

109 be prevented or delayed. A pharmacological agonist of $A B C A 1$ has been shown to restore $A P O E 4$

110 lipidation and ameliorates A $\beta 42 /$ Tau pathologies in a mouse model of human APOE expression

111 (Boehm-Cagan et al., 2016a; Hafiane et al., 2015). Thus, evidence is mounting that lipids are

112 inextricably linked with pathogenic mechanisms in AD and other neurodegenerative diseases

113 (Chung et al., 2020; Griendling et al., 2016; Lin et al., 2019; Reed, 2011).

114 Here, we demonstrate that the fly homologs of multiple genes identified in AD GWAS and

115 other clinical studies (PICALM, CD2AP, AP2A2, ABCA1, ABCA7, LRP1, VPS26, and VPS35) play 
116 a role in the formation of glial LDs, providing a mechanism by which these AD risk genes are

117 involved in neuronal dysfunction. We demonstrate that two ABCA transporters are required in

118 neurons, presumably for the export of lipids, and that six genes, involved in the uptake of APOE,

119 are required in glia for the formation of glial LDs. Our data also show that elevated ROS synergizes

120 with $\mathrm{A} \beta$ production to accelerate neuronal death in flies and exacerbates amyloid plaque formation

121 in mice. Finally, we show that a peptide, previously shown to enhance the lipid-binding properties

122 of $A P O E 4$, restores glial LD formation in a humanized fly model of APOE4. These data place AD-

123 risk genes in a functional pathway that connects ROS with lipid production and A $\beta$ toxicity, thus

124 providing a possible therapeutic avenue for clinical intervention of AD based on utilization of 125 antioxidants that can cross the blood-brain-barrier (BBB).

127 Results

128 ABCA transporters are required in neurons for glial LD formation.

As we have previously shown that apolipoproteins are required for the transfer of peroxidated lipids from neurons to glia, we hypothesized that additional proteins are necessary for

131 the export of lipids from neurons to the extracellular space where they lipidate apolipoproteins.

132 Lipoproteins would then bind to a receptor on glial cells where endocytosis leads to the trafficking

133 of the lipoproteins-rich endosomes to the lysosome for degradation of proteins and subsequent

134 transport of the lipids to the ER for LD formation. We set out to assess the role of AD risk135 associated proteins that export lipids from cells that are also expressed in neurons (Abuznait and 136 Kaddoumi, 2012; Pereira et al., 2017).

137 The adenosine triphosphate-binding cassette transporter A1 (ABCA1) and A7 (ABCA7) 138 encode lipid floppases that transfer lipids from the inner leaflet to the outer leaflet of the cell 
membrane making them available for export to APOE acceptor particles (Tarling et al., 2013;

140 Turton and Morgan, 2013; Wahrle et al., 2004). Further, nonsense variants in both $A B C A 1$ and

$141 A B C A 7$ have been associated with increased risk of developing AD (Bossche et al., 2016; Chang

142 et al., 2019; Chen et al., 2016; Fehér et al., 2018; Teresa et al., 2020). ABCA7 has also been

143 identified as an AD risk locus in GWAS and is known to facilitate clearance of amyloid- $\beta$ (Aikawa

144 et al., 2018). Thus, the ABCA transporters are attractive candidates for neuronal export of lipids

145 induced by ROS.

We set out to identify the fly homologs of ABCA1 and ABCA7 in the fly nervous system.

147 The fly genome contains 10 putative ABCA transporters compared to 13 ABCA transporters in

148 humans, and pairwise analysis of protein sequence does not reveal an obvious 1:1 homology. To

149 identify fly ABCA proteins that share the greatest homology with ABCA1 and ABCA7 we

150 assembled a gene tree of human and fly ABCA protein sequences. We found that fly genes

151 CG34120 and Eato grouped with human ABCA1/2/4/7/12/13 suggesting that these two fly genes

152 may be orthologs of this group of human transporters (Figure 2A).

To assess whether ABCA transporters function in glial lipid droplet formation in the

154 presence of neuronal ROS we used RNAi mediated knockdown to reduce ABCA expression in

155 glia and neurons. As in previous studies, we utilized a ROS-induced LD fly model in which ROS

156 production is initiated in photoreceptor neurons via $R h$-mediated expression of an RNAi targeting

157 the mitochondrial complex I gene, ND42 (Liu et al., 2015). This induces LD formation in

158 surrounding pigment glia that can be visualized, after staining with Nile Red, via confocal

159 microscopy (Figure 2B). In this background, we induced expression of RNAi targeting all the fly

160 ABCA genes for which RNAi is effective, in either neurons, using $R h-G A L 4$, or glia, using $54 C$ -

161 GAL4 and stained for LD. We quantified the efficiency of RNAi-mediated knockdown of each 
162 ABCA gene targeted in our analysis. We found that RNAi constructs targeting Eato, CG34120,

$163 C G 8908, C G 31213, C G 1494$, and $A B C A 3$ all efficiently reduced the expression of their respective

164 ABCA transporters (Figure S1A). We observed significantly reduced LD formation when Eato

165 and $C G 34120$, but not $C G 8908, C G 31213, C G 1494$, or $A B C A 3$ were knocked down in

166 photoreceptors with two independent RNAi lines (Figure 2C-E, data not shown) compared to a

167 control RNAi (luciferase RNAi). In contrast, when these genes were downregulated in glia, no

168 obvious reduction in LD formation was observed (Figure $2 \mathrm{~F}-\mathrm{H}$ ). These data demonstrate that two

169 fly ABCA transporters, Eato and CG34120, are required in photoreceptor neurons for glial LD

170 formation.

Our previous data showed a neuroprotective role for glial LD formation under ROS (Liu

172 et al., 2015, 2017). Hence, we assessed if loss of LD formation, caused by RNAi targeting Eato

173 and CG34120, could lead to an age-dependent neurodegeneration. We tested whether neuronal or

174 glial knockdown of Eato and CG34120 leads to increased neuronal dysfunction, under ROS

175 conditions induced by Rh:ND42 IR, using the electroretinogram (ERG) assay. ERGs serve as a

176 functional readout of neuronal function and viability (Jaiswal et al., 2012). ERG amplitudes were

177 quantified in young (5 days post eclosion) and aged (20 days post eclosion) flies expressing

178 RNAi targeting Eato, CG34120, or a control gene, Luciferase (luc). We observed a significant

179 reduction in ERG amplitude with age when Eato and CG34120 were targeted by RNAi in

180 neurons (Figure $2 \mathrm{~K}-\mathrm{L}$, quantified in M-N) arguing that these two ABCA transporters are

181 required in neurons for the neuroprotective mechanism of glial sequestration of peroxidated

182 lipids in LD.

183 In summary, these data support a role for Eato and CG34120, orthologs of the AD risk

184 genes $A B C A 1$ and $A B C A 7$, in lipid transfer. Both genes are required in neurons, but not glia, for 
glial LD formation, supporting that they mediate the export of peroxidated lipids from neurons to glia to protect neurons from the toxic effects of lipid peroxidation.

The APOE receptor, LRP1, and the retromer are required for glial LD formation

Previous reports demonstrated that glial LD formation requires the fly apolipoprotein,

190 Glaz, supporting the idea that once lipids are exported across the cell membrane of neurons, they

191 bind to apolipoproteins in the extracellular space and are taken up by glia (Liu et al., 2017). Similar

192 conclusions were derived from experiments with primary cultures of vertebrate neurons and glia

193 showing a requirement for APOE for LD formation in glia (Ioannou et al., 2019b; Liu et al., 2017).

194 In these experiments, the endocytosis inhibitor Pitstop2 completely blocked lipid transfer from

195 neurons to glia. However it remained unclear whether the inhibition of endocytosis acts on neurons

196 or glia. We therefore assessed the effects of reduced neuronal or glial expression of genes involved

197 in receptor-mediated endocytosis on glial LD formation, beginning with the genes encoding

198 previously defined apolipoprotein receptors, LRP1 and VLDLR (fly LpR2) (Herz, 2009; Lane-

199 Donovan and Herz, 2017; Rodríguez-Vázquez et al., 2015). RNAi targeting these genes were

200 expressed in photoreceptor neurons (Rh-GAL4) or pigment glia (54C-GAL4), as before, to assess

201 impacts on glial LD formation when ROS is induced in neurons. Loss of LRP1 in glia, but not

202 neurons, caused a significant reduction in LD formation (Figure 3A and E). In contrast, neither

203 neuronal nor glial expression of $L p R 2$ RNAi, altered LD formation (Figure $3 \mathrm{~B}$ and F). These data

204 argue that lipids produced under neuronal ROS require LRPI in glia, specifically.

We then performed ERGs to assess if loss of LRPI in glia could impact age-dependent

206 photoreceptor loss in animals with elevated levels of ROS in neurons. We found that, compared to

207 control flies, glial, but not neuronal, knockdown of LRP1 caused reduced ERG amplitudes in an 
age-dependent manner, indicative of photoreceptor degeneration (Figure 3K-N). These data

209 suggest that the apolipoprotein receptor, LRP1, mediates glial import of peroxidated lipids

210 produced in neurons and promotes glial LD formation and neuroprotection. These data also

211 implicate receptor-mediated endocytosis as a critical pathway in glial LD formation. We therefore

212 hypothesized that LRP1 recycling and vesicle endocytosis would similarly be important for LD

213 formation.

214 The retromer serves critical functions in endocytosis, receptor recycling, and its loss in

215 both photoreceptors and glia leads to neurodegeneration 20 days (Wang et al., 2014). Moreover,

216 the retromer has been linked to several neurodegenerative diseases including AD (Berman et al.,

217 2015; Muhammad et al., 2008) and deficiency of the retromer complex or its cargo proteins impairs

218 endosomal trafficking of amyloid precursor protein (APP), resulting in the overproduction of $\beta$ -

219 amyloid (A $\beta$ ) (Qureshi et al., 2020; Zhang et al., 2018).

To determine the function of the retromer in LD formation, we targeted the retromer genes

221 VPS26 and VPS35 with RNAi to knockdown their expression in our neuronal ROS model. Vps26

222 and Vps35 RNAi were expressed in neurons or glia and we assayed for LD formation and ERG

223 amplitude. We found that loss of Vps26 and Vps35 in either neurons or glia leads to a significant

224 reduction in glial LDs suggesting that the retromer is required in both neurons and glia for LD

225 formation (Figure 3C-D and G-H). We assayed for age-dependent photoreceptor degeneration via

226 ERG and found no worsening of photoreceptor function over time when Vps26 or Vps35 were

227 knocked down in neurons (Figure 3K and M). In contrast, knockdown of Vps26 and Vps35 in glia

228 caused an age-dependent reduction in amplitude indicative of neurodegeneration (Figure 3L and

$229 \mathrm{~N})$. These data suggest that the neurodegeneration observed when glial retromer is lost may be

230 caused by reduced uptake of peroxidated lipids in glia due to reduced apolipoprotein receptor 
231 recycling (Dhawan et al., 2020). The absence of ERG defects when expression of these genes is

232 reduced in neurons at the time points assayed suggests that ROS production or the response to

233 ROS production in neurons is blunted or delayed as the amplitude is decreased by 20 days, but is

234 not statistically significantly different. However, the severe loss of ERGs documented in Wang et

235 al. (2014) when either the Vps26 or Vps35 proteins is lost in both photoreceptors and glia suggest

236 and additive or synergistic effect between neurons an glia and argues that the retromer is required

237 in both cell types to maintain neuronal health.

239 Endocytic AD-risk genes are required in glia for glial LD formation

240 A subset of AD-risk loci contain genes involved in endocytosis, including BIN1, CD2AP,

241 PICALM, AP2A2, and RIN3 (Van Acker et al., 2019; Nelson et al., 2020; Shen et al., 2020). This

242 suggests that disruptions of this process may be important for AD pathogenesis. It is typically

243 thought that these genes contribute to AD pathology through their well characterized function in

244 synaptic transmission in neurons (Gan and Watanabe, 2018; Kaksonen and Roux, 2018; Seto et

245 al., 2002; Takei and Haucke, 2001). However, we hypothesized that these endocytic genes also

246 play a role in LD biogenesis by endocytosing lipids secreted from neurons for LD formation.

248 formation and ERG phenotypes in animals in which homologs of AD-risk genes are targeted, via

249 RNAi, in neurons and glia in the presence of ND42 knockdown-mediated neuronal ROS induction.

250 We found that knockdown of $\operatorname{cindr}(C D 2 A P), A p-2 \alpha(A P 2 A 2)$, and lap (PICALM) in glia, but not

251 neurons, caused a reduction in LD formation (Figure 4A-D, G-J), thus implicating these genes as

252 critical components of glial LD production. In contrast, reduced expression of spri (RIN3) and

253 amph (BIN1) in neurons or glia did not affect LD production (Figure 4E-F, K-L). We observed an 
254 age-dependent decrease in ERG amplitude for the LD-critical genes Ap-2 $\alpha$, lap, and cindr when

255 these were targeted in glia, suggesting that loss of these genes in glia promotes neurodegeneration

256 due to failure to produce neuroprotective LDs in glia (Figure 4O-R). Taken together, these data

257 suggest that loss or reduction of endocytosis in glia inhibits the neuroprotective effects of glial LD

258 formation and implicates endocytosis in the process of neurodegeneration.

259 We next investigated whether clathrin-mediated endocytosis is required for the uptake of 260 neuron-derived fatty acids in a mammalian cell culture system. Since knockdown of lap in glia

261 (Figure 4) caused a significant reduction in LD formation, we chose to knockdown the mammalian

262 homologue PICALM in astrocytes and test the effects of fatty acid transport. We used lentivirus to

263 deliver three independent shRNAs to reduce PICALM protein compared to a non-targeting shRNA

264 control in cultured astrocytes (Figure 5A and B). We incubated neurons with a fluorescently

265 labelled fatty acid analog Red-C12 overnight and then co-cultured the labeled neurons with

266 transduced astrocytes on different coverslips separated by paraffin wax (Figure 5C) (Ioannou et

267 al., 2019b, 2019a). We found a significant reduction in the transfer of fluorescently labelled fatty

268 acids to astrocytes when PICALM levels are reduced (Figure 5D and E). Note that even relatively

269 minor reductions of PICALM in astrocytes lead to reduced lipid accumulations. These data show

270 that clathrin-mediated endocytosis is critical for the internalization of neuron-derived fatty acids

271 in a mammalian culture system.

272

\section{Amyloid Beta synergizes with ROS in flies and mice}

274 In $\mathrm{AD}$, the plaque protein Amyloid $\beta$ has lipophilic properties and has been shown to

275 bind to the apolipoprotein receptor, LRP1, suggesting that altered lipid transfer may also alter

276 amyloid deposition (Ermondi et al., 2015; Moreira et al., 2007; Verghese et al., 2013). There is 
277 also growing evidence that poorly lipidated APOE aggregates and acts as a seed for A $\beta$ plaques

278 (Lanfranco et al., 2020; Sharman et al., 2010; Verghese et al., 2013). This is supported by

279 findings that ABCA1 loss-of-function leads to decreased APOE lipidation and increased

280 amyloidogenesis (Koldamova et al., 2005; Wahrle et al., 2008). Further, as ROS-induced glial

281 LD formation seems to be controlled by AD-associated risk genes and that peroxidated lipids

282 accumulate in pre-AD patients (Allan Butterfield and Boyd-Kimball, 2018; Bradley-Whitman

283 and Lovell, 2015; Bradley et al., 2012; Peña-Bautista et al., 2019), we hypothesized that ROS-

284 induced lipid peroxidation may alter the effects of amyloid deposition in our model.

285 To test this hypothesis we expressed a secreted form of human $A \beta 42$ in photoreceptor

286 neurons, via $R h$-Gal4 (Chouhan et al., 2016). Low levels of ROS production, that avoids

287 substantial neurotoxicity, was induced in animals by feeding them $25 \mu \mathrm{M}$ rotenone. Animals that

288 expressed A $\beta 42$ alone, or were fed rotenone alone, do not exhibit obvious signs of neuronal

289 death in the fly retina and only a small number of glial LDs were observed in either condition

290 (Figure 6A-B, D-E). In contrast, when A $\beta 42$-expressing animals were fed $25 \mu \mathrm{M}$ of rotenone, by

29110 days robust glial LD accumulation and a severe loss of rhabdomeres and overall PR

292 morphology were observed (Figure 6F). These data demonstrate that extracellular A $\beta 42$ strongly

293 synergizes with low levels of ROS to induce premature neuronal death providing a mechanistic

294 link between A $\beta 42$ clearing and ROS.

We next tested for synergism between ROS and amyloid in a vertebrate model using the

296 well-characterized 5XFAD mouse model (Jackson Labs) that expresses APP and forms A $\beta$ -

297 plaques by 4 months of age (Oakley et al., 2006). Previous studies have demonstrated that ROS

298 can be induced in mice by rearing animals in a hyperoxic environment (Ferrari et al., 2017). We

299 assembled cohorts of heterozygous 5XFAD mice and wild-type littermate controls and subjected 
them to either hyperoxia $\left(55 \% \mathrm{O}_{2}\right)$ or normoxia $\left(\sim 21 \% \mathrm{O}_{2}\right)$ conditions for 3 months beginning at

301 the age of 4 weeks. Sagittal brain sections of the mice were probed for A $\beta$ using established

302 immunohistochemistry techniques (Sillitoe et al., 2008) (Figure 6G-J). We quantified plaque

303 number and size in three regions of the brain, namely the cortex, hippocampus, and hindbrain. In

304 each of these regions, plaque size and number observed in 5XFAD mice was significantly

305 elevated in hyperoxia when compared to normoxia (Figure 6K-L, data not shown). Hence, ROS

306 exacerbates $A \beta$-dependent phenotypes in mice similar to $A \beta 42$ expressing flies.

A pharmacological ABCA agonist peptide rescues APOE4 phenotypes

We previously reported that the AD-associated APOE4 allele was much less capable of

310 mediating the transfer of lipids from neurons to glia (Liu et al., 2017). This work used APOE

311 alleles to replace the fly apolipoprotein Glaz by inserting a T2A-GAL4 sequence into the Glaz

312 Gene. This allele can drive the expression of any UAS transgene in the same spatiotemporal pattern

313 as Glaz (Lee et al., 2018). Because we found that ABCA1 may be a critical lipid exporter in

314 neurons exposed to ROS (see Fig. 2) and ABCA1 agonist peptides can promote APOE4 activity

315 in AD mice (Boehm-Cagan et al., 2016a; Hafiane et al., 2015), we hypothesized that

316 pharmacologically enhancing ABCA1 activity may restore LD formation in APOE4 flies.

We generated a fly line that expresses a genetically encoded version of the ABCA1 agonist

318 peptide that was previously identified (Boehm-Cagan et al., 2016a, 2016b; Hafiane et al., 2015).

319 The peptide sequence was cloned downstream of an Argos secretion signal to enable peptide

320 release from the cell. Expression of the peptide was driven by the Glaz $^{\text {T2A-Gal4 }}$ allele. We then

321 elevated ROS levels in these animals by expressing an RNAi against Marf, the fly homolog of

322 Mitofusin, under control of the $R h$ promoter, similar to Rh-ND42 IR as above (Liu et al., 2017). 
323 We confirmed the previous report that heterozygous Glaz ${ }^{\text {T2A-Gal4 }}$ animals have significantly

324 reduced LD production (Liu et al., 2017) and showed that expression of the peptide does not alter

325 LD formation in this background (Figure 7A and E). We next co-expressed the peptide with

326 APOE2, APOE3 or APOE4 alleles and compared it with expression of the APOE alleles alone

327 (Figure 7B-D and F-J). Peptide expression with either APOE2 or APOE3 does not alter LD

328 production in the absence of peptide expression. In contrast, expression of the peptide with APOE4

329 restored LD formation, suggesting that the peptide can promote the lipidation of APOE4 in flies

330 and restore glial LD formation. These data show that this peptide indeed modifies the function of

331 APOE4 and restore its activity but has no impact on APOE2 and APOE3.

\section{Discussion}

We show that glia act to buffer against ROS produced in neurons by taking up peroxidated

335 lipids and sequestering them in LDs (Figure 8). We found that glial sequestration of peroxidated 336 lipids into LDs is neuroprotective and requires the function of genes involved in lipid export 337 (ABCA1 and $A B C A 7)$, lipid capture and transport (APOE) (Ioannou et al., 2019b; Liu et al., 2017), 338 and receptor-mediated endocytosis (LRP1, VPS26, VPS35, PICALM, CD2AP and AP2A2).

339 Notably, these genes have been implicated in AD and other neurodegenerative disorder risk genes

340 (Hafiane et al., 2015; Kunkle et al., 2019; Lambert et al., 2013; Muhammad et al., 2008; Shinohara

341 et al., 2017). These data suggest that AD risk could be elevated in the presence of partial loss-of-

342 function variants of genetic risk factors by inducing lower efficiency of lipid transfer and 343 peroxidated lipid sequestration in glial LD. This model predicts that the cumulative risk conferred 344 by variants in the process of neuron-to-glia lipid transfer lies in the non-cell autonomous responses 345 to neuronal ROS. Affected glia will be less well equipped to sequester peroxidated lipids, leading 
to increased levels of peroxidated lipids within and surrounding neurons, thus exacerbating

347 neuronal demise. Although glia are well-equipped to sequester peroxidated lipids, they have a

348 limited capacity to do so. Glia eventually succumb to the adverse effects of peroxidated lipid

349 storage which eventually lead to a subsequent loss of neurons. It has been well documented that

350 ROS levels are elevated with age and in multiple neurodegenerative diseases, including AD, and

351 may be an important underlying cause of disease-associated neurodegeneration (Singh et al.,

352 2019). Neurons have limited antioxidant capacity and innate mechanisms to respond to increased

353 ROS by activating cellular responses (Burnside and Hardingham, 2017). Developing a better

354 understanding of how protection against ROS is carried out should inform us about new ways to

355 protect the nervous system from oxidative insult. Furthermore, understanding how these responses

356 go awry may reveal ways to exogenously potentiate the antioxidant response. The identification

357 of multiple genetic risk factors for $\mathrm{AD}$ that mediate glial LD formation (Figures 2-4), in

358 combination with the observations that $A \beta$ may be exacerbated by low levels of ROS (Figure 6)

359 suggest that ROS-induced neuronal peroxidated lipid production and transfer to glia constitutes an

360 important facet of the antioxidant toolkit (Liu et al., 2015, 2017). These data are also consistent

361 with the previous observation that astrocytes are markedly more robust in handling and detoxifying

362 ROS (Burnside and Hardingham, 2017). Hence, we argue that there is a critical need to reduce

363 ROS in neurons in aging and the context of disease. We previously found that the fatty acid

364 transporter protein, Fatp, is required in LD formation (Liu et al., 2017). However, it is not known

365 to export lipids across membranes. Instead, Fatp may interact with lipid transport proteins such as

366 ABCA transporters, known to transport lipids including cholesterol, phospholipids, fatty acids as

367 well as other lipids across membranes (Neumann et al., 2017; Tarling et al., 2013). ABCA7, has

368 been demonstrated to assemble high-density lipoprotein particles (Abe-Dohmae et al., 2004; 
Hayashi et al., 2005) and implicated in AD pathogenesis, first in an Icelandic population (Steinberg et al., 2015) and, later, in a larger AD cohort (Kunkle et al., 2019). The ABCA1 N1800H mutation,

371 which has a low prevalence, is increased in frequency in the AD population and is associated with

372 hemorrhagic stroke, consistent with clinical presentations in APOE4 carriers (Nordestgaard et al.,

373 2015). Interestingly, the sequence of the ABCA1 agonist CS6253 restored LD formation in APOE4

374 flies but did not affect APOE2 or APOE3 function (Figure 7), supporting the findings of AD

375 prevention in APOE4 targeted replacement mice (Boehm-Cagan et al., 2016a). This, together with

376 our findings that $\mathrm{ABCA}$ transporters in the fly are required in neurons for glial LD formation

377 (likely by mediating the export of peroxidated lipids) suggest a critical role for ABCA genes in

378 proper lipid regulation in disease.

The genes implicated in endocytosis studied in this work have often been studied in the context of synaptic transmission and/or neurodegenerative disease. BINI is a membrane fission

381 protein that regulates endocytic vesicle size in vertebrates, but it has been implicated in APP 382 processing as well as Tau degradation (Van Acker et al., 2019; Ramjaun et al., 1997; Takeda et 383 al., 2018). The fly homolog, Amphiphysin (Amph), regulates transverse tubule formation in 384 muscles, which was also shown to be affected in vertebrate mutants (Lee et al., 2002) but Amph 385 has not been implicated in endocytosis in flies to our knowledge (Razzaq et al., 2001; Zelhof et 386 al., 2001). In contrast, $C D 2 A P$ is a scaffolding protein that has been implicated in endocytosis and 387 vesicle trafficking as well as APP sorting and processing in vertebrates (Furusawa et al., 2019; 388 Harrison et al., 2016; Ubelmann et al., 2017). However, severe loss of function alleles of the fly 389 homolog, cindr, affects synapse maturation as well as synaptic vesicle recycling and release 390 (Ojelade et al., 2019). PICALM is a clathrin assembly protein that has been implicated in the import 391 of $\gamma$-secretase and APP processing as well as Tau buildup (Van Acker et al., 2019; Baig et al., 
2010). The fly homolog, like-AP180 (lap) acts as a clathrin adaptor to promote clathrin-coated

393 vesicle formation and restrict coated vesicle size as well as the efficacy of synaptic vesicle protein

394 retrieval (Zhang et al., 1998). AP2A2, a member of the AP-2 adaptor protein complex, which aids

395 in assembling endocytic components in flies and vertebrates, has implications in AD risk

396 (González-Gaitán and Jäckle, 1997; Nelson et al., 2020). Finally, RIN3 is a Rab5 guanine

397 nucleotide exchange factor important for recruiting CD2AP and BIN1 to endosomes and has been

398 implicated in APP accumulation and Tau phosphorylation regulation (Shen et al., 2020). Based on

399 our data, three of these genes (CD2AP, AP2A2, and PICALM) play critical functions in glia for

400 LD formation (Figure 4). Historically, because many of the endocytic AD-risk genes are known

401 to play a critical role in synaptic transmission, it is thought that their role in AD pathology may

402 occur due to loss of function of these genes in neurons. However, single cell RNA sequencing

403 databases provide evidence for enriched expression of many of the endocytic AD risk genes in

404 mouse/human glia, including those targeted in this study (Zhang et al., 2014, 2016) and our data

405 show that glia are highly sensitive to partial loss of these genes as exemplified in Figure 5 for

406 PICALM.

407 As endocytic vesicles are processed in the cell, the retromer is critical for protein recycling

408 including cell surface receptors and rhodopsin (Wang et al., 2014). We observed reduced LD

409 formation when retromer function was targeted via RNAi in both neurons and glia (Figure 3).

410 However, neurodegeneration was observed when the retromer was lost in glia, but not neurons.

411 This suggests a different role for the retromer in neurons and glia. In glia, the LRP1 receptor is

412 critical for LD formation and the retromer is required for LRP1 recycling back to the membrane

413 for efficient uptake of lipid particles (Stockinger et al., 2002). It also plays a critical role in Tau

414 spreading (Rauch et al., 2020). Hence, loss of LD due to retromer dysfunction in glia would lead 
415 to LD loss and neurodegeneration, consistent with our observations. In mice CS6253 increased

416 LRP1 in the hippocampus of APOE4 mice but it did not affect APOE3 mice which is not

417 inconsistent with our data. Loss of retromer in neurons, may also lead to a progressive

418 neurodegeneration but neurons may be less sensitive to this loss, as knockdown of $\operatorname{Vps} 26$ in both

419 glia and neurons causes more severe neuron loss than does knockdown of Vps26 in glia alone

420 (Wang et al., 2014; this study). Follow-up studies are needed to distinguish these hypotheses, but

421 it is becoming increasingly evident that the retromer plays critical roles in the maintenance of

422 neurons in AD (Muhammad et al., 2008).

423 Our model predicts that as ROS levels rise with age or other environmental factors, it

424 becomes more difficult for glia to sequester peroxidated lipids, which promotes

425 neurodegeneration. Thus, while approaches to induce uptake of lipids to remove ROS and amyloid

426 from neurons or the extracellular space, it's important to consider additional approaches to

427 neutralize ROS early in disease to prevent glial death and eventual neurodegeneration. Our model

428 also helps explain the non-linear relationship between amyloid burden and clinical severity of

429 disease. Human A $\beta$ expression induces neurodegeneration in Drosophila (Chouhan et al., 2016)

430 and neurological and behavioral phenotypes in mice (Kobayashi and Chen, 2005; Oakley et al.,

431 2006). Notably, production of low levels of ROS or A $\beta$ alone causes neurotoxicity in a very slow

432 and progressive manner. In flies, overexpression of $A \beta 42$ causes neuronal death after

433 approximately 45 days (Chouhan et al., 2016). However, we found that combining low amounts

434 of ROS in A $\beta 42$-expressing flies strongly exacerbated neurodegeneration and enhanced $A \beta$

435 deposition in 5XFAD mice (Figure 6). It is noteworthy that $\mathrm{A} \beta$ and peroxidated lipids both bind

436 APOE (Allan Butterfield et al., 2002; Lanfranco et al., 2020), providing a possible mechanism of

437 ROS/A 342 synergy. Importantly, APOE4 is not properly lipidated and lipidation of APOE4 is 
required for Aß42 binding (Kanekiyo et al., 2014; Namba et al., 1991; Strittmatter et al., 1993).

439 Hence, APOE4 is unable to properly clear peroxidated lipids as well as A $\beta 42$, strongly accelerating

440 the demise of neurons. High amyloid burden may induce severe disease only in the presence of

441 genetic or environmental triggers that induce ROS.

Regardless of the cause of ROS (e.g. age, environmental stress, or genetic perturbations),

443 oxidative stress may initiate disease in an individual with a previous genetic predisposition to

444 disease (APOE4, or other genetic disease risk). This model predicts that a reduction of oxidative

445 stress, regardless of the cause, may help mitigate damage done by peroxidated lipids, and prevent

446 further lipid biogenesis in neurons. Our model also suggest that the risk genes identified in GWAS

447 that are involved in lipid handling and endocytosis may affect transport of peroxidated lipids from

448 neurons into glia. Indeed, antioxidant levels are altered in AD patients and the use of antioxidants

449 as a treatment for AD has been proposed previously, although with mixed outcomes (Frank and

450 Gupta, 2005; Mullan et al., 2017; Vina et al., 2011; Wojtunik-Kulesza et al., 2016). Hence, animal

451 models that better recapitulate AD phenotypes should consider the use of a combination of genetic

452 manipulation and environmental ROS.

453 Numerous mammalian models have been generated to model AD that induce amyloid

454 and/or tau production in the brain. Despite clear differences between the etiology and risk factors

455 associated with the development of FAD and sporadic AD, many research groups model disease

456 in animals using dominant FAD-causing mutations. This is because both forms of AD are

457 histopathologically and clinically similar and because sporadic AD cannot be easily modelled in

458 animals (LaFerla and Green, 2012). One historically common choice for mouse models of AD

459 research is the 5XFAD mouse which harbors three mutations in the APP locus and two mutations

460 in PS1 (Oakley et al., 2006). Each of these five mutations, on their own, are FAD-causing 
mutations in humans (Jankowsky and Zheng, 2017). Although this mouse line exhibits a very high

462 amyloid burden, it almost certainly does not provide the most relevant model for human disease.

463 While these mammalian models have proven fruitful for understanding many aspects of AD, none

464 of them is adequate in reproducing the entirety of symptoms manifested in AD. We argue that the

465 efficacy in disease modeling in mammals may be improved by the addition of ROS which is largely

466 absent in the AD field currently. We acknowledge that the addition of ROS in mammalian models

467 comes with various challenges including the use of toxic drugs (i.e. rotenone) or bulky and

468 expensive equipment (i.e. hyperoxic animal chambers). Genetic mutations that induce ROS may

469 be a more viable option to include in the background of AD models. A study using a mouse model

470 of Leigh Syndrome in which the gene NDUFS4 is knocked out, thereby reducing activity of

471 Complex I and leading to elevated ROS production results in early death of the Ndufs $4^{-{ }_{-}}$animals

472 (Assouline et al., 2012; Quintana et al., 2012). These mice have numerous LD in astrocytes and

473 microglia prior to the onset of neuronal loss (Liu et al., 2015). In hypoxia these mice live much

474 longer than when reared in normoxic conditions (Jain et al., 2016). Thus, the addition of ROS via

475 genetic means, by for example removing a copy of $N d u f s 4$ may prove a viable method to induce

476 ROS in an otherwise monogenic AD mammalian model.

Although age and mitochondrial dysfunction are obvious sources of ROS in AD patients,

478 there may be numerous other conditions that induce ROS production and subsequent lipid

479 peroxidation, LD formation, and eventual neurodegeneration. A careful examination of ROS in

480 AD patients and inclusion of ROS in animal models may help begin to provide mechanistic insight

481 into the etiology and progression of this complex disease. We argue that the use of antioxidants

482 that penetrate the blood-brain barrier requires further investigation and that these treatments may

483 aid prevention of neuron loss as observed in flies (Liu et al., 2015). 


\section{References}

A. Alzheimer (1911). On certain peculiar diseases of old age. Clin. Anat. 8, 429-431. Abe-Dohmae, S., Ikeda, Y., Matsuo, M., Hayashi, M., Okuhira, K.I., Ueda, K., and Yokoyama, S. (2004). Human ABCA7 Supports Apolipoprotein-mediated Release of Cellular Cholesterol and Phospholipid to Generate High Density Lipoprotein. J. Biol. Chem. 279, 604-611. Abuznait, A.H., and Kaddoumi, A. (2012). Role of ABC transporters in the pathogenesis of Alzheimers disease. ACS Chem. Neurosci. 3, 820-831. Van Acker, Z.P., Bretou, M., and Annaert, W. (2019). Endo-lysosomal dysregulations and lateonset Alzheimer's disease: Impact of genetic risk factors. Mol. Neurodegener. 14, 1-20. Aikawa, T., Holm, M.L., and Kanekiyo, T. (2018). ABCA7 and pathogenic pathways of Alzheimer's disease. Brain Sci. 8, 1-13.

Allan Butterfield, D., and Boyd-Kimball, D. (2018). Oxidative Stress, Amyloid- $\beta$ Peptide, and Altered Key Molecular Pathways in the Pathogenesis and Progression of Alzheimer's Disease. J. Alzheimer's Dis. 62, 1345-1367.

Allan Butterfield, D., Castegna, A., Lauderback, C.M., and Drake, J. (2002). Evidence that amyloid beta-peptide-induced lipid peroxidation and its sequelae in Alzheimer's disease brain contribute to neuronal death. Neurobiol. Aging 23, 655-664.

Alzheimer, A. (1907). Uber eine eigenartige Erkrankung der Hirnrinde. Allg. Zeitschrift Rsychiatrie Psych. Medizine 64, 146-148. Andreone, B.J., Przybyla, L., Llapashtica, C., Rana, A., Davis, S.S., van Lengerich, B., Lin, K., Shi, J., Mei, Y., Astarita, G., et al. (2020). Alzheimer's-associated PLC $\gamma 2$ is a signaling node required for both TREM2 function and the inflammatory response in human microglia. Nat. Neurosci. 23, 927-938.

Assouline, Z., Jambou, M., Rio, M., Bole-Feysot, C., de Lonlay, P., Barnerias, C., Desguerre, I., Bonnemains, C., Guillermet, C., Steffann, J., et al. (2012). A constant and similar assembly defect of mitochondrial respiratory chain complex I allows rapid identification of NDUFS4 mutations in patients with Leigh syndrome. 1822, 1062-1069.

Baig, S., Joseph, S.A., Tayler, H., Abraham, R., Owen, M.J., Williams, J., Kehoe, P.G., and Love, S. (2010). Distribution and expression of picalm in alzheimer disease. J. Neuropathol. Exp. Neurol. 69, 1071-1077. Barish, S., Nuss, S., Strunilin, I., Bao, S., Mukherjee, S., Jones, C.D., and Volkan, P.C. (2018). Combinations of DIPs and Dprs control organization of olfactory receptor neuron terminals in Drosophila. PLoS Genet. 14, 1-33.

Beaudoin, G.M.J., Lee, S.H., Singh, D., Yuan, Y., Ng, Y.G., Reichardt, L.F., and Arikkath, J. (2012). Culturing pyramidal neurons from the early postnatal mouse hippocampus and cortex. Nat. Protoc. 7, 1741-1754.

Behl, C. (1997). Amyloid $\beta$-protein toxicity and oxidative stress in Alzheimer's disease. Cell Tissue Res. 290, 471-480.

Berbée, J.F.P., Mol, I.M., Milne, G.L., Pollock, E., Hoeke, G., Lütjohann, D., Monaco, C., Rensen, P.C.N., van der Ploeg, L.H.T., and Shchepinov, M.S. (2017). Deuterium-reinforced polyunsaturated fatty acids protect against atherosclerosis by lowering lipid peroxidation and hypercholesterolemia. Atherosclerosis 264, 100-107.

Berman, D.E., Ringe, D., Petsko, G.A., and Small, S.A. (2015). The Use of Pharmacological Retromer Chaperones in Alzheimer's Disease and other Endosomal-related Disorders. Neurotherapeutics 12, 12-18. 
Bielicki, J.K. (2016). ABCA1 agonist peptides for the treatment of disease. Curr. Opin. Lipidol. 27, 40-46.

Bischof, J., Björklund, M., Furger, E., Schertel, C., Taipale, J., and Basler, K. (2012). A versatile platform for creating a comprehensive UAS-ORFeome library in Drosophila. Dev. 140, 2434 2442. Alzheimer's disease: Roles of microglia-mediated synaptic remodeling, inflammation, and oxidative stress. Neurobiol. Stress 9, 9-21.

Bloom, G.S. (2014). Amyloid- $\beta$ and tau: The trigger and bullet in Alzheimer disease

540 Boehm-Cagan, A., and Michaelson, D.M. (2014). Reversal of apoE4-Driven Brain Pathology and Behavioral Deficits by Bexarotene. J. Neurosci. 34, 7293-7301. Boehm-Cagan, A., Bar, R., Liraz, O., Bielicki, J.K., Johansson, J.O., and Michaelson, D.M. (2016a). ABCA1 Agonist Reverses the ApoE4-Driven Cognitive and Brain Pathologies. J. Alzheimer's Dis. 54, 1219-1233.

Boehm-Cagan, A., Bar, R., Harats, D., Shaish, A., Levkovitz, H., Bielicki, J.K., Johansson, J.O., and Michaelson, D.M. (2016b). Differential Effects of apoE4 and Activation of ABCA1 on Brain and Plasma Lipoproteins. PLoS One 11, 1-17.

Bossche, T. Van Den, Sleegers, K., Cuyvers, E., Engelborghs, S., Sieben, A., Roeck, A. De, Cauwenberghe, C. Van, Vermeulen, S., Broeck, M. Van Den, Laureys, A., et al. (2016).

550 Phenotypic characteristics of Alzheimer patients carrying an ABCA7 mutation. Neurology 86,

551 2126-2133.

Bradley-Whitman, M.A., and Lovell, M.A. (2015). Biomarkers of lipid peroxidation in Alzheimer disease (AD): an update. Arch. Toxicol. 89, 1035-1044. Bradley, M.A., Xiong-Fister, S., Markesbery, W.R., and Lovell, M.A. (2012). Elevated 4hydroxyhexenal in Alzheimer's disease (AD) progression. Neurobiol. Aging 33, 1034-1044. Van Den Brink, D.M., Cubizolle, A., Chatelain, G., Davoust, N., Girard, V., Johansen, S., Napoletano, F., Dourlen, P., Guillou, L., Angebault-Prouteau, C., et al. (2018). Physiological and pathological roles of FATP-mediated lipid droplets in Drosophila and mice retina. PLOS Genet. $14,1-25$.

560 Burnside, S.W., and Hardingham, G.E. (2017). Transcriptional regulators of redox balance and other homeostatic processes with the potential to alter neurodegenerative disease trajectory. Butterfield, D.A. (2020). Brain lipid peroxidation and alzheimer disease: Synergy between the Butterfield and Mattson laboratories. Ageing Res. Rev. 64, 1568-1637. Chang, Y.T., Hsu, S.W., Huang, S.H., Huang, C.W., Chang, W.N., Lien, C.Y., Lee, J.J., Lee, C.C., and Chang, C.C. (2019). ABCA7 polymorphisms correlate with memory impairment and default mode network in patients with APOEє4-associated Alzheimer's disease. Alzheimer's Res. Ther. 11, 103-113.

Chen, Q., Liang, B., Wang, Z., Cheng, X., Huang, Y., Liu, Y., and Huang, Z. (2016). Influence of four polymorphisms in ABCA1 and PTGS2 genes on risk of Alzheimer's disease: a metaanalysis. Neurol. Sci. 37, 1209-1220.

Chouhan, A.K., Guo, C., Hsieh, Y.-C.C., Ye, H., Senturk, M., Zuo, Z., Li, Y., Chatterjee, S., Botas, J., Jackson, G.R., et al. (2016). Uncoupling neuronal death and dysfunction in Drosophila models of neurodegenerative disease. Acta Neuropathol. Commun. 4, 62-76.

574 Chung, H. lok, Wangler, M.F., Marcogliese, P.C., Jo, J., Ravenscroft, T.A., Zuo, Z., Duraine, L., 575 Sadeghzadeh, S., Li-Kroeger, D., Schmidt, R.E., et al. (2020). Loss- or Gain-of-Function 
Mutations in ACOX1 Cause Axonal Loss via Different Mechanisms. Neuron 106, 589-606.e6. Conejero-Goldberg, C., Gomar, J.J., Bobes-Bascaran, T., Hyde, T.M., Kleinman, J.E., Herman, M.M., Chen, S., Davies, P., and Goldberg, T.E. (2014). APOE2 enhances neuroprotection against alzheimer's disease through multiple molecular mechanisms. Mol. Psychiatry 19, 12431250 .

581 Dhawan, K., Naslavsky, N., Caplan, S., and Hanson, P.I. (2020). Sorting nexin 17 (SNX17) links endosomal sorting to Eps 15 homology domain protein 1 (EHD1)-mediated fission machinery. J.

583 Biol. Chem. 295, 3837-3850.

584 Ermondi, G., Catalano, F., Vallaro, M., Ermondi, I., Leal, M.P.C., Rinaldi, L., Visentin, S., and promote hydrophobic and electrostatic interactions. Int. J. Pharm. 495, 179-185.

587 Fan, J., Donkin, J., and Wellington, C. (2009). Greasing the wheels of A $\beta$ clearance in Alzheimer's Disease: The role of lipids and apolipoprotein e. BioFactors 35, 239-248. Fehér, Á., Giricz, Z., Juhász, A., Pákáski, M., Janka, Z., and Kálmán, J. (2018). ABCA1 rs2230805 and rs2230806 common gene variants are associated with Alzheimer's disease.

591 Neurosci. Lett. 664, 79-83.

592 Ferrari, M., Jain, I.H., Goldberger, O., Rezoagli, E., Thoonen, R., Cheng, K.-H., Sosnovik, D.E., 593 Scherrer-Crosbie, M., Mootha, V.K., and Zapol, W.M. (2017). Hypoxia treatment reverses neurodegenerative disease in a mouse model of Leigh syndrome. Proc. Natl. Acad. Sci. U. S. A. 114, E4241-E4250.

596 Frank, B., and Gupta, S. (2005). A review of antioxidants and Alzheimer's disease. Ann. Clin.

597 Psychiatry 17, 269-286.

598 Furusawa, K., Takasugi, T., Chiu, Y.W., Hori, Y., Tomita, T., Fukuda, M., and Hisanaga, S. ichi 599 (2019). CD2-associated protein (CD2AP) overexpression accelerates amyloid precursor protein 600 (APP) transfer from early endosomes to the lysosomal degradation pathway. J. Biol. Chem. 294, $601 \quad 10886-10899$.

602 Gan, Q., and Watanabe, S. (2018). Synaptic vesicle endocytosis in different model systems.

603 Front. Cell. Neurosci. 12, 171-197.

604 Giau, V. Van, Bagyinszky, E., Yang, Y.S., Youn, Y.C., An, S.S.A., and Kim, S.Y. (2019).

605 Genetic analyses of early-onset Alzheimer's disease using next generation sequencing. Sci. Rep.

$6069,1-10$.

607 González-Gaitán, M., and Jäckle, H. (1997). Role of drosophila $\alpha$-adaptin in presynaptic vesicle 608 recycling. Cell 88, 767-776.

609 Götz, J., Deters, N., Doldissen, A., Bokhari, L., Ke, Y., Wiesner, A., Schonrock, N., and Ittner, 610 L.M. (2007). A decade of tau transgenic animal models and beyond. In Brain Pathology, (John 611 Wiley \& Sons, Ltd), pp. 91-103.

612 Götz, J., Eckert, A., Matamales, M., Ittner, L.M., and Liu, X. (2011). Modes of A $\beta$ toxicity in 613 Alzheimer's disease. Cell. Mol. Life Sci. 68, 3359-3375.

614 Griendling, K.K., Touyz, R.M., Zweier, J.L., Dikalov, S., Chilian, W., Chen, Y.R., Harrison, 615 D.G., and Bhatnagar, A. (2016). Measurement of Reactive Oxygen Species, Reactive Nitrogen 616 Species, and Redox-Dependent Signaling in the Cardiovascular System: A Scientific Statement 617 from the American Heart Association. Circ. Res. 119, e39-e75.

618 Grimm, A., and Eckert, A. (2017). Brain aging and neurodegeneration: from a mitochondrial 619 point of view. J. Neurochem. 143, 418-431.

620 Hafiane, A., Bielicki, J.K., Johansson, J.O., and Genest, J. (2015). Novel apo E-derived ABCA1 621 agonist peptide (CS-6253) promotes reverse cholesterol transport and induces formation of pre $\beta$ - 
1 HDL in vitro. PLoS One $10,1-32$.

623 Hardy, J., Selkoe, D.J., Ovod, V., Munsell, L., Kasten, T., Morris, J.C., Yarasheski, K.E., and Bateman, R.J. (2002). The amyloid hypothesis of Alzheimer's disease: progress and problems on the road to therapeutics. Science 297, 353-356. Harrison, B.J., Venkat, G., Lamb, J.L., Hutson, T.H., Drury, C., Rau, K.K., Bunge, M.B., Mendell, L.M., Gage, F.H., Johnson, R.D., et al. (2016). The adaptor protein CD2AP is a coordinator of neurotrophin signaling-mediated axon arbor plasticity. J. Neurosci. 36, 42594275.

Hatters, D.M., Peters-Libeu, C.A., and Weisgraber, K.H. (2006). Apolipoprotein E structure: insights into function. Trends Biochem. Sci. 31, 445-454. of high density lipoprotein generated by ABCA1 and ABCA7. J. Lipid Res. 46, 1703-1711. Heisenberg, M. (1971). Separation of Receptor and Lamina Potentials in the Electroretinogram of Normal and Mutant Drosophila. J. Exp. Biol. 55, 85 LP - 100. Herz, J. (2009). Apolipoprotein E receptors in the nervous system. Curr. Opin. Lipidol. 20, 190196.

Huang, Y., and Mahley, R.W. (2014). Apolipoprotein E: Structure and function in lipid metabolism, neurobiology, and Alzheimer's diseases. Neurobiol. Dis. 72, 3-12. Huynh, T.-P.V.P. V., Davis, A.A., Ulrich, J.D., and Holtzman, D.M. (2017). Apolipoprotein E and Alzheimer's disease: the influence of apolipoprotein E on amyloid-? and other amyloidogenic proteins. 58, 824-836. Ioannou, M.S., Jackson, J., Sheu, S.-H., Chang, C.-L., Weigel, A. V., Liu, H., Pasolli, H.A., Xu, C.S., Pang, S., Matthies, D., et al. (2019a). Neuron-Astrocyte Metabolic Coupling Protects against Activity-Induced Fatty Acid Toxicity. Cell 177, 1522-1535.e14. Ioannou, M.S., Liu, Z., and Lippincott-Schwartz, J. (2019b). A Neuron-Glia Co-culture System for Studying Intercellular Lipid Transport. Curr. Protoc. Cell Biol. 84, 1-21. Jain, I.H., Zazzeron, L., Goli, R., Alexa, K., Schatzman-Bone, S., Dhillon, H., Goldberger, O., Peng, J., Shalem, O., Sanjana, N.E., et al. (2016). Hypoxia as a therapy for mitochondrial disease. Science (80-. ). 352, 54-61. Jaiswal, M., Sandoval, H., Zhang, K., Bayat, V., and Bellen, H.J. (2012). Probing mechanisms that underlie human neurodegenerative diseases in Drosophila. Annu. Rev. Genet. 46, 371-396. Jankowsky, J.L., and Zheng, H. (2017). Practical considerations for choosing a mouse model of Alzheimer's disease. Mol. Neurodegener. 12, 89-110. Kaksonen, M., and Roux, A. (2018). Mechanisms of clathrin-mediated endocytosis. Nat. Rev. Mol. Cell Biol. 19, 313-326. Kanekiyo, T., Xu, H., and Bu, G. (2014). ApoE and A $\beta$ in Alzheimer's disease: Accidental encounters or partners? Neuron 81, 740-754. Karch, C.M., and Goate, A.M. (2015). Alzheimer's disease risk genes and mechanisms of disease pathogenesis. Biol. Psychiatry 77, 43-51.

Kobayashi, D.T., and Chen, K.S. (2005). Behavioral phenotypes of amyloid-based genetically modified mouse models of Alzheimer's disease. Genes, Brain Behav. 4, 173-196.

Koldamova, R., Staufenbiel, M., and Lefterov, I. (2005). Lack of ABCA1 considerably decreases brain ApoE level and increases amyloid deposition in APP23 mice. J. Biol. Chem. 280, 4322443235.

Kumar, S., Stecher, G., Li, M., Knyaz, C., and Tamura, K. (2018). MEGA X: Molecular evolutionary genetics analysis across computing platforms. Mol. Biol. Evol. 35, 1547-1549. 
Kunkle, B.W., Grenier-Boley, B., Sims, R., Bis, J.C., Damotte, V., Naj, A.C., Boland, A., Vronskaya, M., van der Lee, S.J., Amlie-Wolf, A., et al. (2019). Genetic meta-analysis of

670 diagnosed Alzheimer's disease identifies new risk loci and implicates $A \beta$, tau, immunity and lipid processing. Nat. Genet. 51, 414-430. LaFerla, F.M., and Green, K.N. (2012). Animal models of Alzheimer disease. Cold Spring Harb. Perspect. Med. 2, 1-13. Lambert, J.-C., Ibrahim-Verbaas, C.A., Harold, D., Naj, A.C., Sims, R., Bellenguez, C., Jun, G., DeStefano, A.L., Bis, J.C., Beecham, G.W., et al. (2013). Meta-analysis of 74,046 individuals identifies 11 new susceptibility loci for Alzheimer's disease. Nat. Genet. 45, 1452-1458. Lane-Donovan, C., and Herz, J. (2017). ApoE, ApoE Receptors, and the Synapse in Alzheimer's Disease. Trends Endocrinol. Metab. 28, 273-284. Lanfranco, M.F., Ng, C.A., and Rebeck, G.W. (2020). ApoE lipidation as a therapeutic target in Alzheimer's disease. Int. J. Mol. Sci. 21, 1-19. Lee, E., Marcucci, M., Daniell, L., Pypaert, M., Weisz, O.A., Ochoa, G.C., Farsad, K., Wenk, M.R., and De Camilli, P. (2002). Amphiphysin 2 (Bin1) and T-tubule biogenesis in muscle. Science (80-. ). 297, 1193-1196. Lee, P.T., Zirin, J., Kanca, O., Lin, W.W., Schulze, K.L., Li-Kroeger, D., Tao, R., Devereaux, C., Hu, Y., Chung, V., et al. (2018). A gene-specific T2A-GAL4 library for drosophila. Elife 7. Li, Z., Shue, F., Zhao, N., Shinohara, M., and Bu, G. (2020). APOE2: protective mechanism and therapeutic implications for Alzheimer's disease. Mol. Neurodegener. 15, 63-81.

688 Lin, G., Wang, L., Marcogliese, P.C., and Bellen, H.J. (2019). Sphingolipids in the Pathogenesis of Parkinson's Disease and Parkinsonism. Trends Endocrinol. Metab. 30, 106-117. Liu, L., Zhang, K., Sandoval, H., Yamamoto, S., Jaiswal, M., Sanz, E., Li, Z., Hui, J., Graham, B.H., Quintana, A., et al. (2015). Glial Lipid Droplets and ROS Induced by Mitochondrial Defects Promote Neurodegeneration. Cell 160, 177-190.

Liu, L., MacKenzie, K.R., Putluri, N., Maletić-Savatić, M., and Bellen, H.J. (2017). The GliaNeuron Lactate Shuttle and Elevated ROS Promote Lipid Synthesis in Neurons and Lipid Droplet Accumulation in Glia via APOE/D. Cell Metab. 26, 719-737.e6.

Marioni, R.E., Harris, S.E., Zhang, Q., McRae, A.F., Hagenaars, S.P., Hill, W.D., Davies, G., Ritchie, C.W., Gale, C.R., Starr, J.M., et al. (2018). GWAS on family history of Alzheimer's disease. Transl. Psychiatry 8, 1-7.

Masdeu, J.C. (2020). Neuroimaging of Diseases Causing Dementia. Neurol. Clin. 38, 65-94. Michaelson, D.M. (2014). APOE $\varepsilon 4$ : The most prevalent yet understudied risk factor for Alzheimer's disease. Alzheimer's Dement. 10, 861-868. Protein Modifications and Interactions: Their Role in Function and Dysfunction. Int. J. Mol. Sci. 15, 4671-4713.

Moreira, P.I., Nunomura, A., Honda, K., Aliev, G., Casadesus, G., Zhu, X., Smith, M.A., and Perry, G. (2007). The key role of oxidative stress in alzheimer's disease. In Oxidative Stress and Neurodegenerative Disorders, (Elsevier), pp. 267-281. Muhammad, A., Flores, I., Zhang, H., Yu, R., Staniszewski, A., Planel, E., Herman, M., Ho, L., Kreber, R., Honig, L.S., et al. (2008). Retromer deficiency observed in Alzheimer's disease causes hippocampal dysfunction, neurodegeneration, and $\mathrm{A} \beta$ accumulation. Proc. Natl. Acad. Sci. U. S. A. 105, 7327-7332. 
are altered in Alzheimer's disease: A case-control study. Alzheimer's Dement. Transl. Res. Clin. Interv. 3, 432-439.

Namba, Y., Tomonaga, M., Kawasaki, H., Otomo, E., and Ikeda, K. (1991). Apolipoprotein E immunoreactivity in cerebral amyloid deposits and neurofibrillary tangles in Alzheimer's disease and kuru plaque amyloid in Creutzfeldt-Jakob disease. Brain Res. 541, 163-166. Nelson, P.T., Fardo, D.W., and Katsumata, Y. (2020). The MUC6/AP2A2 Locus and Its Relevance to Alzheimer's Disease: A Review. J. Neuropathol. Exp. Neurol. 79, 568-584. Neumann, J., Rose-Sperling, D., and Hellmich, U.A. (2017). Diverse relations between ABC transporters and lipids: An overview. Biochim. Biophys. Acta - Biomembr. 1859, 605-618. Nordestgaard, L.T., Tybjærg-Hansen, A., Nordestgaard, B.G., and Frikke-Schmidt, R. (2015). Loss-of-function mutation in ABCA1 and risk of Alzheimer's disease and cerebrovascular disease. Alzheimer's Dement. 11, 1430-1438.

Nugent, A.A., Lin, K., van Lengerich, B., Lianoglou, S., Przybyla, L., Davis, S.S., Llapashtica, C., Wang, J., Kim, D.J., Xia, D., et al. (2020). TREM2 Regulates Microglial Cholesterol Metabolism upon Chronic Phagocytic Challenge. Neuron 105, 837-854.e9. Oakley, H., Cole, S.L., Logan, S., Maus, E., Shao, P., Craft, J., Guillozet-Bongaarts, A., Ohno, M., Disterhoft, J., Van Eldik, L., et al. (2006). Intraneuronal beta-Amyloid Aggregates, Neurodegeneration, and Neuron Loss in Transgenic Mice with Five Familial Alzheimer's 10140. Ojelade, S.A., Lee, T. V., Giagtzoglou, N., Yu, L., Ugur, B., Li, Y., Duraine, L., Zuo, Z., Petyuk, V., De Jager, P.L., et al. (2019). cindr, the Drosophila Homolog of the CD2AP Alzheimer's Disease Risk Gene, Is Required for Synaptic Transmission and Proteostasis. Cell Rep. 28, 17991813.e5. Di Paolo, G., and Kim, T.-W. (2011). Linking lipids to Alzheimer's disease: cholesterol and beyond. Nat. Rev. Neurosci. 12, 284-296. Peña-Bautista, C., López-Cuevas, R., Cuevas, A., Baquero, M., and Cháfer-Pericás, C. (2019). Lipid peroxidation biomarkers correlation with medial temporal atrophy in early Alzheimer Disease. Neurochem. Int. 129, 104519.

Pereira, C.D., Martins, F., Wiltfang, J., Da Cruz E Silva, O.A.B., and Rebelo, S. (2017). ABC Transporters Are Key Players in Alzheimer's Disease. J. Alzheimer's Dis. 61, 463-485. Quintana, A., Zanella, S., Koch, H., Kruse, S.E., Lee, D., Ramirez, J.M., and Palmiter, R.D. (2012). Fatal breathing dysfunction in a mouse model of Leigh syndrome. 122, 2359-2368. Qureshi, Y.H., Baez, P., and Reitz, C. (2020). Endosomal Trafficking in Alzheimer's Disease, Parkinson's Disease, and Neuronal Ceroid Lipofuscinosis. Mol. Cell. Biol. 40, 1-12.

Ramjaun, A.R., Micheva, K.D., Bouchelet, I., and McPherson, P.S. (1997). Identification and characterization of a nerve terminal-enriched amphiphysin isoform. J. Biol. Chem. 272, 1670016706.

Rauch, J.N., Luna, G., Guzman, E., Audouard, M., Challis, C., Sibih, Y.E., Leshuk, C., Hernandez, I., Wegmann, S., Hyman, B.T., et al. (2020). LRP1 is a master regulator of tau uptake and spread. Nature 580, 381-385.

755 Razzaq, A., Robinson, I.M., McMahon, H.T., Skepper, J.N., Su, Y., Zelhof, A.C., Jackson, A.P., 756 Gay, N.J., and O'Kane, C.J. (2001). Amphiphysin is necessary for organization of the excitationcontraction coupling machinery of muscles, but not for synaptic vesicle endocytosis in Drosophila. Genes Dev. 15, 2967-2979.

Reed, T.T. (2011). Lipid peroxidation and neurodegenerative disease. Free Radic. Biol. Med. 51, 
1302-1319.

Regen, F., Hellmann-Regen, J., Costantini, E., and Reale, M. (2017). Neuroinflammation and Alzheimer's Disease: Implications for Microglial Activation. Curr. Alzheimer Res. 14. Ristow, M., and Schmeisser, S. (2011). Extending life span by increasing oxidative stress. Free Radic. Biol. Med. 51, 327-336. Robert, J., Button, E.B., Yuen, B., Gilmour, M., Kang, K., Bahrabadi, A., Stukas, S., Zhao, W., Kulic, I., and Wellington, C.L. (2017). Clearance of beta-amyloid is facilitated by apolipoprotein E and circulating highdensity lipoproteins in bioengineered human vessels. Elife 6, 1-24. Rodríguez-Vázquez, M., Vaquero, D., Parra-Peralbo, E., Mejía-Morales, J.E., and Culi, J. (2015). Drosophila Lipophorin Receptors Recruit the Lipoprotein LTP to the Plasma Membrane to Mediate Lipid Uptake. PLOS Genet. 11, 1-24.

Rogaeva, E., Kawarai, T., and St. George-Hyslop, P. (2006). Genetic complexity of Alzheimer's disease: Successes and challenges. J. Alzheimer's Dis. 9, 381-387.

Schindelin, J., Arganda-Carreras, I., Frise, E., Kaynig, V., Longair, M., Pietzsch, T., Preibisch, S., Rueden, C., Saalfeld, S., Schmid, B., et al. (2012). Fiji: An open-source platform for biological-image analysis. Nat. Methods 9, 676-682.

Seto, E.S., Bellen, H.J., and Lloyd, T.E. (2002). When cell biology meets development: Endocytic regulation of signaling pathways. Genes Dev. 16, 1314-1336.

Sharman, M.J., Morici, M., Hone, E., Berger, T., Taddei, K., Martins, I.J., Lim, W.L.F., Singh, S., Wenk, M.R., Ghiso, J., et al. (2010). APOE genotype results in differential effects on the peripheral clearance of amyloid- $\beta 42$ in APOE knock-in and knock-out mice. J. Alzheimer's Dis. 21, 403-409. Shen, R., Zhao, X., He, L., Ding, Y., Xu, W., Lin, S., Fang, S., Yang, W., Sung, K., Spencer, B., et al. (2020). Upregulation of RIN3 induces endosomal dysfunction in Alzheimer's disease.

Transl. Neurodegener. 9, 26-44.

Shinohara, M., Tachibana, M., Kanekiyo, T., and Bu, G. (2017). Role of LRP1 in the pathogenesis of Alzheimer's disease: Evidence from clinical and preclinical studies. J. Lipid Res. 58, 1267-1281.

Sillitoe, R. V., Stephen, D., Lao, Z., and Joyner, A.L. (2008). Engrailed homeobox genes determine the organization of Purkinje cell sagittal stripe gene expression in the adult cerebellum. J. Neurosci. 28, 12150-12162. Singh, A., Kukreti, R., Saso, L., and Kukreti, S. (2019). Oxidative stress: A key modulator in neurodegenerative diseases. Molecules 24, 1-20.

Steinberg, S., Stefansson, H., Jonsson, T., Johannsdottir, H., Ingason, A., Helgason, H., Sulem, P., Magnusson, O.T., Gudjonsson, S.A., Unnsteinsdottir, U., et al. (2015). Loss-of-function variants in ABCA7 confer risk of Alzheimer's disease. Nat. Genet. 47, 445-447.

Stelzmann, R.A., Norman Schnitzlein, H., and Reed Murtagh, F. (1995). An english translation of alzheimer's 1907 paper, “über eine eigenartige erkankung der hirnrinde.” Clin. Anat. 8, 429431. Nimpf, J. (2002). The PX-domain protein SNX17 interacts with members of the LDL receptor family and modulates endocytosis of the LDL receptor. EMBO J. 21, 4259-4267.

803 Strittmatter, W.J., Saunders, A.M., Schmechel, D., Pericak-Vance, M., Enghild, J., Salvesen, G.S., and Roses, A.D. (1993). Apolipoprotein E: High-avidity binding to $\beta$-amyloid and increased frequency of type 4 allele in late-onset familial Alzheimer disease. Proc. Natl. Acad. Sci. U. S. A. 90, 1977-1981. 
De Strooper, B., and Karran, E. (2016). The Cellular Phase of Alzheimer's Disease. Cell 164, 603-615.

808 Takeda, T., Kozai, T., Yang, H., Ishikuro, D., Seyama, K., Kumagai, Y., Abe, T., Yamada, H., Uchihashi, T., Ando, T., et al. (2018). Dynamic clustering of dynamin-amphiphysin helices regulates membrane constriction and fission coupled with GTP hydrolysis. Elife 7, 1-19. Takei, K., and Haucke, V. (2001). Clathrin-mediated endocytosis: Membrane factors pull the trigger. Trends Cell Biol. 11, 385-391. Tarling, E.J., Vallim, T.Q. d. A., and Edwards, P.A. (2013). Role of ABC transporters in lipid transport and human disease. Trends Endocrinol. Metab. 24, 342-350. Teresa, J.C., Fernado, C., Nancy, M.R., Gilberto, V.A., Alberto, C.R., and Roberto, R.R. (2020). Association of genetic variants of ABCA1 with susceptibility to dementia: (SADEM study). Metab. Brain Dis. 35, 915-922. Thapa, A., and Carroll, N.J. (2017). Dietary modulation of oxidative stress in Alzheimer's disease. Int. J. Mol. Sci. 18, 1583-1596. Tönnies, E., and Trushina, E. (2017). Oxidative Stress, Synaptic Dysfunction, and Alzheimer's Disease. J. Alzheimer's Dis. 57, 1105-1121.

Turton, J., and Morgan, K. (2013). ATP-binding cassette, subfamily A (ABC1), member 7 (ABCA7). In Genetic Variants in Alzheimer's Disease, (Springer New York), pp. 135-158. Ubelmann, F., Burrinha, T., Salavessa, L., Gomes, R., Ferreira, C., Moreno, N., and Guimas Almeida, C. (2017). Bin1 and CD 2 AP polarise the endocytic generation of beta-amyloid. EMBO Rep. 18, 102-122. Venken, K.J.T., He, Y., Hoskins, R.A., and Bellen, H.J. (2006). P[acman]: A BAC transgenic platform for targeted insertion of large DNA fragments in D. melanogaster. Science (80-. ). 314, 1747-1751.

830 Verghese, P.B., Castellano, J.M., Garai, K., Wang, Y., Jiang, H., Shah, A., Bu, G., Frieden, C., and Holtzman, D.M. (2013). ApoE influences amyloid- $\beta$ (A $\beta$ ) clearance despite minimal apoE/A $\beta$ association in physiological conditions. Proc. Natl. Acad. Sci. U. S. A. 110, E1807E1816. Cao, Y., Roos, J., and Bellen, H.J. (2003). Synaptojanin is recruited by endophilin to promote synaptic vesicle uncoating. Neuron 40, 733-748. Vina, J., LLoret, A., Giraldo, E., C. Badia, M., and D. Alonso, M. (2011). Antioxidant Pathways in Alzheimers Disease: Possibilities of Intervention. Curr. Pharm. Des. 17, 3861-3864. Wahrle, S.E., Jiang, H., Parsadanian, M., Legleiter, J., Han, X., Fryer, J.D., Kowalewski, T., and Holtzman, D.M. (2004). ABCA1 is required for normal central nervous system apoE levels and for lipidation of astrocyte-secreted apoE. J. Biol. Chem. 279, 40987-40993. Wahrle, S.E., Jiang, H., Parsadanian, M., Kim, J., Li, A., Knoten, A., Jain, S., HirschReinshagen, V., Wellington, C.L., Bales, K.R., et al. (2008). Overexpression of ABCA1 reduces amyloid deposition in the PDAPP mouse model of Alzheimer disease. J. Clin. Invest. 118, 671682.

Wang, Y., and Mandelkow, E. (2016). Tau in physiology and pathology. Nat. Rev. Neurosci. 17, 5-21.

850 Wang, S., Tan, K.L., Agosto, M.A., Xiong, B., Yamamoto, S., Sandoval, H., Jaiswal, M., Bayat, V., Zhang, K., Charng, W.L., et al. (2014). The Retromer Complex Is Required for Rhodopsin Recycling and Its Loss Leads to Photoreceptor Degeneration. PLoS Biol. 12, 1-20. Wang, Y., Cella, M., Mallinson, K., Ulrich, J.D., Young, K.L., Robinette, M.L., Gilfillan, S., 
Krishnan, G.M., Sudhakar, S., Zinselmeyer, B.H., et al. (2015). TREM2 lipid sensing sustains the microglial response in an Alzheimer's disease model. Cell 160, 1061-1071. Ward, A., Crean, S., Mercaldi, C.J., Collins, J.M., Boyd, D., Cook, M.N., and Arrighi, H.M. (2012). Prevalence of Apolipoprotein E4 Genotype and Homozygotes (APOE e4/4) among Patients Diagnosed with Alzheimer's Disease: A Systematic Review and Meta-Analysis. Neuroepidemiology 38, 1-17.

858 Wellington, C. (2004). Cholesterol at the crossroads: Alzheimer's disease and lipid metabolism.

859 Clin. Genet. 66, 1-16.

860 Wojtunik-Kulesza, K.A., Oniszczuk, A., Oniszczuk, T., and Waksmundzka-Hajnos, M. (2016).

861 The influence of common free radicals and antioxidants on development of Alzheimer's Disease.

862 Biomed. Pharmacother. 78, 39-49.

863 Wong, M.W., Braidy, N., Poljak, A., Pickford, R., Thambisetty, M., and Sachdev, P.S. (2017). Dysregulation of lipids in Alzheimer's disease and their role as potential biomarkers.

866 Zabel, M., Nackenoff, A., Kirsch, W.M., Harrison, F.E., Perry, G., and Schrag, M. (2018).

867 Markers of oxidative damage to lipids, nucleic acids and proteins and antioxidant enzymes

868 activities in Alzheimer's disease brain: A meta-analysis in human pathological specimens. Free

869 Radic. Biol. Med. 115, 351-360.

870 Zelhof, A.C., Bao, H., Hardy, R.W., Razzaq, A., Zhang, B., and Doe, C.Q. (2001). Drosophila

871 Amphiphysin is implicated in protein localization and membrane morphogenesis but not in

872 synaptic vesicle endocytosis. Development 128, 5005-5015.

873 Zhang, C., and Liu, P. (2017). The lipid droplet: A conserved cellular organelle. Protein Cell 1-

8745.

875 Zhang, B., Koh, Y.H., Beckstead, R.B., Budnik, V., Ganetzky, B., and Bellen, H.J. (1998).

876 Synaptic vesicle size and number are regulated by a clathrin adaptor protein required for 877 endocytosis. Neuron 21, 1465-1475.

878 Zhang, H., Huang, T., Hong, Y., Yang, W., Zhang, X., Luo, H., Xu, H., and Wang, X. (2018).

879 The retromer complex and sorting nexins in neurodegenerative diseases. Front. Aging Neurosci.

$88010,1-11$.

881 Zhang, Y., Chen, K., Sloan, S.A., Bennett, M.L., Scholze, A.R., O’Keeffe, S., Phatnani, H.P., 882 Guarnieri, P., Caneda, C., Ruderisch, N., et al. (2014). An RNA-sequencing transcriptome and 883 splicing database of glia, neurons, and vascular cells of the cerebral cortex. J. Neurosci. 34,

884 11929-11947.

885 Zhang, Y., Sloan, S.A., Clarke, L.E., Caneda, C., Plaza, C.A., Blumenthal, P.D., Vogel, H., 886 Steinberg, G.K., Edwards, M.S.B.B., Li, G., et al. (2016). Purification and Characterization of 887 Progenitor and Mature Human Astrocytes Reveals Transcriptional and Functional Differences 888 with Mouse Highlights. Neuron 89, 37-53.

889 Zhu, X.C., Tan, L., Wang, H.F., Jiang, T., Cao, L., Wang, C., Wang, J., Tan, C.C., Meng, X.F., 890 and Yu, J.T. (2015). Rate of early onset Alzheimer's disease: A systematic review and meta891 analysis. Ann. Transl. Med. 3, 38-43. 


\section{Acknowledgements}

894 We are grateful to the Bloomington Drosophila Stock Center and the Vienna Drosophila Resource Center for providing reagents. 5XFAD mice were provided to us through a gift of Huda Zoghbi. Mouse pathological studies were carried out by the Cell and Tissue Pathogenesis Core which is supported by the Eunice Kennedy Shriver National Institute of Child Health \& Human Development of the National Institutes of Health (NIH) under the award number P50HD103555. Confocal images were captured in the Neurovisualization Core of the Intellectual and Developmental Disabilities Research Center (IDDRC), which is supported by the NIH under the award number U54HD083092. This work was supported by a grant from the Texas Alzheimer's Research and Care Consortium under the award number 2018-05-11-II, to H.J.B. M.J.M. was supported by the Medical Genetics Research Fellowship Training Grant from the NIH under the award number T32 GM07526-41. L.D.G. was supported by the Brain Disorders \& Development Fellowship Training Grant from the NIH under the award number T32 NS04312418. P.C.M. is supported by a grant from Canadian Institutes of Health Research under the award number MFE-164712. M.S.I. is supported by the Canadian Institutes of Health Research under the award number 173321, and the Heart \& Stroke Foundation of Canada under the award number 170722. I.R. is supported by the Alberta Synergies in Alzheimer's and Related Disorders (SynAD) program funded by the Alzheimer Society of Alberta and Northwest Territories through their Hope for Tomorrow program and the University Hospital Foundation. SynAD operates in partnership with the Neuroscience and Mental Health Institute at the University of Alberta. H.J.B. is an investigator of the Howard Hughes Medical Institute (HHMI) and thanks

\section{Author Contributions} J.G.H., and H.B.; Writing - Review \& Editing, M.J.M., S.B., L.D.G., P.C.M., J.G.H., M.S.I, and H.J.B.; Supervision, H.J.B.; Funding Acquisition, H.J.B.

\section{Declaration of Interests}

J.O.J. is the President and CEO of Artery Therapeutics, Inc.

\section{Star Methods \\ Resource Availability \\ Lead Contact}

Further information and requests for resources and reagents should be directed to and will be fulfilled by the Lead Contact, Hugo Bellen (hbellen@bcm.edu).

Materials Availability

The peptide-expressing fly stock generated in this study was generated with permission through a collaboration with J.O.J and Artery Therapeutics, Inc. All stocks and reagents are freely available upon request to H.J.B.

Data and Code Availability

936 


\section{Experimental Model and Subject Details}

\section{Animals}

Drosophila melanogaster were raised on standard molasses-based lab diet at $22^{\circ} \mathrm{C}$ under

\section{Method Details}

\section{Gene Tree Assembly}

Protein sequences of all human and fly ABCA genes were downloaded from the National Center for Biotechnology Information (NCBI) website. Sequences were aligned using ClustalW algorithm within MEGA X (Kumar et al., 2018). Aligned sequences were used to build the neighbor-joining tree within MEGA X.

\section{Generation of Transgenic Flies}

UAS-ArgosSS::Peptide transgenic flies were generated by ORF synthesis in pUC57 using Drosophila codon-optimized sequence (Integrated DNA Technologies) of the argos secretion signal (MPTTLMLLPCMLLLLLTAAAVAVGG) (Chouhan et al., 2016) upstream of the peptide sequence, where citrulline residues were replaced with arginine residues, (EVRSKLEEWLAALRELAEELLARAKS) (Bielicki, 2016; Boehm-Cagan and Michaelson, 2014). The ORF was shuttled to the Gateway pDONR221 entry vector (ThermoFisher) by BP clonase II reaction (ThermoFisher) using Argos_attB primers. Fully sequence verified clones were shuttled to the pGW-attB-HA destination vector (Bischof et al., 2012) by LR clonase II (ThermoFisher). The UAS construct was inserted into the VK37 (PBac $\{\mathrm{y}[+]-\operatorname{attP}\}$ VK00037) docking site by $\phi \mathrm{C} 31$ mediated transgenesis (Venken et al., 2006).

\section{Lipid Droplet Analysis}

Whole-mount staining of fly retinas with Nile Red to visualize lipids was performed as in Liu, et al. (2015). In brief, fly heads were isolated under PBS and fixed in $3.7 \%$ formaldehyde overnight. Retinas were then dissected under PBS and rinsed three times with 1X PBS and incubated for 20 minutes at 1:1,000 dilution of PBS with $1 \mathrm{mg} / \mathrm{mL}$ Nile Red (Millipore Sigma). Retinas were subsequently rinsed five times with 1X PBS and mounted in Vectashield (Vector Labs) for imaging on a Leica SP8 confocal microscope. Images were obtained using a 63X glycerol submersion lens with $3 \mathrm{X}$ zoom.

\section{Electroretinogram Assay}

Electroretinogram (ERG) assays (Heisenberg, 1971) were performed as previously described (Verstreken et al., 2003). In brief, live flies were immobilized with Elmer's school glue on a microscope slide. Glass electrodes, filled with $3 \mathrm{M} \mathrm{NaCl}$, were placed in the thorax for reference and on the center part of the eye for recording. Prior to recording, flies were maintained in the dark for at least one minute. Approximately one-second light flashes were 
983

984

985

986

987

988

989

990

991

992

993

994

995

996

997

998

999

1000

1001

1002

1003

1004

1005

1006

1007

1008

1009

1010

1011

1012

1013

1014

1015

1016

1017

1018

1019

1020

1021

1022

1023

1024

1025

1026

1027

1028

manually delivered using a halogen lamp. At least three recordings from at least 10 flies per genotype were obtained for analysis using LabChart 8.

\section{Immunohistochemistry}

Animal perfusion, sectioning and immunohistochemistry was performed as in Sillitoe, et al. (2008). In brief, mice were anesthetized with and sacrificed by intracardiac perfusion, initially with saline, followed by $4 \%$ paraformaldehyde in PBS. Mice brains were removed and bisected down the midline with one hemibrain utilized for histopathological analysis, which was immersed again in paraformaldehyde for 24 hours at $4^{\circ} \mathrm{C}$. Hemibrains were then dehydrated and preserved in paraffin. Serial sagittal sections were cut and $6 \mu \mathrm{M}$ sections were mounted on glass slides. After sections were blocked in $10 \%$ normal goat serum and rinsed with PBS, sections were stained with the anti-A $\beta 42$ antibody (Covance, 1:1,000) and a biotinylated anti-mouse IgG antibody (Jackson, 1:200). Slides were then incubated in DAB solution, monitored by eye, and the reaction stopped with distilled water. Finally, slides were counterstained using Mayer's hematoxylin and dehydrated prior to imaging.

\section{qRT-PCR Analysis}

RNA extraction, cDNA synthesis, and qRT-PCR were performed as in Barish et al. ( 2018). In brief, 10 larvae ubiquitously expressing RNAi (via Daugtherless-Gal4) were isolated. RNA extraction was carried out using the RNeasy Mini Kit (Qiagen) followed by reverse transcription into cDNA using iScript Reverse Transcription Supermix (BioRad). Quantitative PCR reactions were performed using iTaq Universal SYBR Green Supermix (BioRad) on a BioRad CFX96 Touch Real-Time PCR Detection System. Three biological and technical replicates were performed for each genotype. Expression $(\mathrm{Ct})$ values were obtained and used to calculate differential expression $(\Delta \mathrm{Ct})$ and normalized to GAPDH expression.

\section{Primary culture of hippocampal neurons and astrocytes}

Hippocampal cultures were generated from P0-P1 Sprague-Dawley rats obtained from Charles River Laboratories that arrived at our facility one week prior to birth. These experiments were approved by the Canadian Council of Animal Care at the University of Alberta (AUP\#3358). Cultures were prepared as previously described (Beaudoin et al., 2012; Ioannou et al., 2019a). In brief, tissue was digested with papain, gently triturated, and filtered with a cell strainer and plated on poly-D-lysine coated coverslips for the transfer assay or plastic tissue culture dishes for Western blot analysis. Neurons were grown in Neurobasal medium containing B-27 supplement, $2 \mathrm{mM}$ Glutamax and antibiotic-antimycotic. Astrocytes were grown in Basal Eagle Media containing $10 \%$ fetal bovine serum, $0.45 \%$ glycose, $1 \mathrm{mM}$ sodium pyruvate, $2 \mathrm{mM}$ Glutamax, and antibioticantimycotic. All cells were grown at $37^{\circ} \mathrm{C}$ in $5 \% \mathrm{CO}_{2}$.

\section{Lentivirus transduction}

Astrocytes at DIV 2 were transduced with SMARTVector lentiviral shRNA (Dharmacon) at an MOI of 3. Three independent shRNA sequences targeting PICALM or a non-targeting control shRNA was used. The media was replaced with fresh culture media after $24 \mathrm{hrs}$. and the cells were used for protein validation or in the transfer assay 5 days later (DIV 7). To validate protein knockdown, astrocytes were lysed in lysis buffer [20 mM HEPES pH 7.4, $100 \mathrm{mM} \mathrm{NaCl,} \%$ Triton X-100, $5 \mathrm{mM}$ EDTA, 1× Halt Protease \& Phosphatase Inhibitor Cocktail (Thermo Scientific)], resolved by SDS-PAGE and processed for Western blotting using anti-PICALM 
rabbit polyclonal (Millipore Sigma) and anti-GAPDH mouse monoclonal (ThermoFisher) as a loading control. Lysates were run in duplicate and statistics were performed on the average of these duplicates for each experiment.

\section{Fatty acid transfer assay}

Neurons (DIV 7) were incubated with $2 \mu$ M BODIPY 558/568 (Red-C12) for 16 hours in neuronal growth media. Neurons were washed twice with warm phosphate-buffered saline (PBS) and incubated with fresh media for 1 hour. Red-C12 labelled neurons and unlabeled astrocytes transduced with lentivirus as described above were washed twice with warm PBS and the coverslips were cultured together (facing each other), separated by paraffin wax and incubated in Hanks' Balanced Salt solution containing calcium and magnesium for 4 hours at $37^{\circ} \mathrm{C}$ (Ioannou et al., 2019b, 2019a). Astrocytes were fixed in 4\% paraformaldehyde, stained with DAPI, and mounted using DAKO fluorescence mounting media. Images were acquired using a Zeiss 710 Laser Scanning Confocal Microscope equipped with a plan-apochromat 63x oil objective (Zeiss, $\mathrm{NA}=1.4)$. Maximum intensity projections of three-dimensional image stacks $(0.5 \mu \mathrm{m}$ sections $)$ of Red-C12 staining were obtained and analyzed using ImageJ. Only astrocytes expressing the lentiviral reporter turboGFP were quantified. Images were thresholded and the number of particles with a pixel size greater than 2 was detected. 10 cells per coverslip were averaged. Schematic of fatty acid transfer assay was created with BioRender.com.

\section{Quantification and Statistical Analysis}

FIJI (Schindelin et al., 2012) was utilized to view fly retinal and mouse brain images and all genotypes were blinded prior to quantification. Lipid droplets with diameter $\geq 0.5 \mu \mathrm{M}$ were manually quantified from fly retinal images. Amyloid plaque number from mouse brain images was manually quantified and amyloid size measurements were taken using the 'Measure' tool in FIJI. LabChart 8 (AD Instruments) was used to view and measure the amplitude of ERG traces. Quantification datasets were assembled in Microsoft Excel 365 for comparison and statistical analysis. For quantification, $\geq 10$ animals per genotype were used. Mean $+/-$ SEM were plotted and pair-wise T-tests were performed with a statistical significance cutoff at $* \mathrm{p}<0.05$, and $* * p<0.01$. Statistical analysis of knockdown efficiency in rat cells used the Kruskal-Wallis test with Dunn's posttest using a significance cutoff at $* p<0.05$. Analysis of lipid transfer utilized One-way ANOVA with Dunnett's posttest using a significance cutoff at $* * * \mathrm{p}<0.001$. 
rabbit polyclonal (Millipore Sigma) and anti-GAPDH mouse monoclonal (ThermoFisher) as a loading control. Lysates were run in duplicate and statistics were performed on the average of these duplicates for each experiment.

Neurons (DIV 7) were incubated with $2 \mu \mathrm{M}$ BODIPY 558/568 (Red-C12) for 16 hours in neuronal growth media. Neurons were washed twice with warm phosphate-buffered saline (PBS) and incubated with fresh media for 1 hour. Red-C12 labelled neurons and unlabeled astrocytes transduced with lentivirus as described above were washed twice with warm PBS and the coverslips were cultured together (facing each other), separated by paraffin wax and incubated in Hanks' Balanced Salt solution containing calcium and magnesium for 4 hours at $37^{\circ} \mathrm{C}$ (Ioannou et al., 2019b, 2019a). Astrocytes were fixed in 4\% paraformaldehyde, stained with DAPI, and mounted using DAKO fluorescence mounting media. Images were acquired using a Zeiss 710 Laser Scanning Confocal Microscope equipped with a plan-apochromat 63x oil objective (Zeiss, $\mathrm{NA}=1.4)$. Maximum intensity projections of three-dimensional image stacks $(0.5 \mu \mathrm{m}$ sections $)$ of Red-C12 staining were obtained and analyzed using ImageJ. Only astrocytes expressing the lentiviral reporter turboGFP were quantified. Images were thresholded and the number of particles with a pixel size greater than 2 was detected. 10 cells per coverslip were averaged. Schematic of fatty acid transfer assay was created with BioRender.com.

\section{Quantification and Statistical Analysis}

FIJI (Schindelin et al., 2012) was utilized to view fly retinal and mouse brain images and all genotypes were blinded prior to quantification. Lipid droplets with diameter $\geq 0.5 \mu \mathrm{M}$ were manually quantified from fly retinal images. Amyloid plaque number from mouse brain images was manually quantified and amyloid size measurements were taken using the 'Measure' tool in FIJI. LabChart 8 (AD Instruments) was used to view and measure the amplitude of ERG traces. Quantification datasets were assembled in Microsoft Excel 365 for comparison and statistical analysis. For quantification, $\geq 10$ animals per genotype were used. Mean $+/-$ SEM were plotted and pair-wise T-tests were performed with a statistical significance cutoff at $* \mathrm{p}<0.05$, and $* * p<0.01$. Statistical analysis of knockdown efficiency in rat cells used the Kruskal-Wallis test with Dunn's posttest using a significance cutoff at $* p<0.05$. Analysis of lipid transfer utilized One-way ANOVA with Dunnett's posttest using a significance cutoff at $* * * p<0.001$. 


\section{KEY RESOURCES TABLE}

\begin{tabular}{|c|c|c|}
\hline REAGENT or RESOURCE & SOURCE & IDENTIFIER \\
\hline \multicolumn{3}{|l|}{ Antibodies } \\
\hline$\overline{\text { Anti } A \beta 42(6 \mathrm{E} 10)}$ & Covance & RRID:AB_662798 \\
\hline Biotinylated anti-mouse lgG & $\begin{array}{l}\text { Jackson Immuno Research } \\
\text { Laboratories, Inc. }\end{array}$ & RRID:AB_2338557 \\
\hline Anti-PICALM rabbit polyclonal & Millipore Sigma & RRID:AB_1855361 \\
\hline Anti-GAPDH mouse monoclonal & ThermoFisher & RRID:AB_2536381 \\
\hline HRP- AffiniPure donkey anti-rabbit & $\begin{array}{l}\text { Jackson Immuno Research } \\
\text { Laboratories, Inc. }\end{array}$ & RRID:AB_2340770 \\
\hline HRP- AffiniPure donkey anti-mouse & $\begin{array}{l}\text { Jackson Immuno Research } \\
\text { Laboratories, Inc. }\end{array}$ & RRID:AB_2340770 \\
\hline \multicolumn{3}{|l|}{ Bacterial and Virus Strains } \\
\hline $\begin{array}{l}\text { SMARTvector hCMV-TurboGFP non-targeting } \\
\text { control particles }\end{array}$ & Dharmacon Inc. & Cat\# S-005000-01 \\
\hline $\begin{array}{l}\text { SMARTvector Lentiviral } \\
\text { Human PICALM hCMV-TurboGFP } \\
\text { shRNA (shRNA1) }\end{array}$ & Dharmacon Inc. & $\begin{array}{l}\text { Cat\# V3SH7590- } \\
225194030\end{array}$ \\
\hline $\begin{array}{l}\text { SMARTvector Lentiviral } \\
\text { Human PICALM hCMV-TurboGFP } \\
\text { shRNA (shRNA2) }\end{array}$ & Dharmacon Inc. & $\begin{array}{l}\text { Cat\# V3SH7590- } \\
225390149\end{array}$ \\
\hline $\begin{array}{l}\text { SMARTvector Lentiviral } \\
\text { Human PICALM hCMV-TurboGFP } \\
\text { shRNA (shRNA3) }\end{array}$ & Dharmacon Inc. & $\begin{array}{l}\text { Cat\# V3SH7590- } \\
225119184\end{array}$ \\
\hline \multicolumn{3}{|l|}{ Chemicals, Peptides, and Recombinant Proteins } \\
\hline Nile Red & Millipore Sigma & Cat \# 72485 \\
\hline Rotenone & Millipore Sigma & Cat \# R8875 \\
\hline $\begin{array}{l}\text { BODIPY 558/568 C12 (4,4-Difluoro-5-(2- } \\
\text { Thienyl)-4-Bora-3a,4a-Diaza-s-Indacene-3- } \\
\text { Dodecanoic Acid) (Red-C12) }\end{array}$ & ThermoFisher Scientific & Cat \# D3835 \\
\hline Vectashield & Vector Labs & Cat \# H-1000-10 \\
\hline 4\% paraformaldehyde & ThermoFisher & Cat \# J61899-AK \\
\hline BP clonase II & ThermoFisher & Cat \# 11789020 \\
\hline LR clonase II & ThermoFisher & Cat \# 11791020 \\
\hline RNeasy Mini Kit & Qiagen & Cat \# 74104 \\
\hline iScript Reverse Transcription Supermix & BioRad & Cat \# 1708840 \\
\hline iTaq Universal SYBR Green Supermix & BioRad & Cat \# 1725120 \\
\hline DAPI & Abcam & Cat\# ab228549 \\
\hline Halt protease \& phosphatase inhibitor cocktail & ThermoFisher & Cat\# 78446 \\
\hline DAKO fluorescence mounting media & $\begin{array}{l}\text { Agilent Technologies Canada } \\
\text { Inc. }\end{array}$ & Cat\# S302380-2 \\
\hline Neurobasal medium & Gibco & Cat\# LS21103049 \\
\hline Basal medium Eagle & Gibco & Cat\# LS21010046 \\
\hline Fetal bovine serum & VWR International Ltd & Cat\# MP97068-085 \\
\hline Sodium pyruvate & Gibco & Cat\# LS11360070 \\
\hline Glutamax & Gibco & Cat\# LS17504044 \\
\hline Antibiotic-antimycotic & Gibco & Cat\# LS15240062 \\
\hline Poly-D-lysine & Millipore Sigma & Cat\# P6407 \\
\hline Papain dissociation enzyme & Worthington Biochemical & Cat\# LK003178 \\
\hline Hanks' Balanced Salt solution & Cytiva Life Sciences & Cat\# SH3026801 \\
\hline
\end{tabular}




\begin{tabular}{|c|c|c|}
\hline \multicolumn{3}{|l|}{ Experimental Models: Organisms/Strains } \\
\hline$y 1 w^{*} ; P\left\{w\left[+m^{*}\right]=G A L 4\right\} 54 C$ & Drosophila melanogaster & BDSC_27328 \\
\hline$P\{N i n a E-G D 6220\}(R h-N D 42$ RNAi) & Drosophila melanogaster & BDSC_76598 \\
\hline$P\{$ NinaE-GD11094\} (Rh-Marf RNAi) & Drosophila melanogaster & BDSC_76597 \\
\hline $\begin{array}{l}\text { y1 w*; PBac\{UAS- } \\
\text { hAPOE.2.C112.C158\}VK00037 }\end{array}$ & Drosophila melanogaster & BDSC_76604 \\
\hline $\begin{array}{l}\text { y1 w*; PBac }\{U A S-A P O E 3 . C 112, \\
R 158\} V K 00037\end{array}$ & Drosophila melanogaster & BDSC_76605 \\
\hline $\begin{array}{l}\text { y1 } w^{*} ; \text { PBac }\{U A S-A P O E 4 . R 112, \\
R 158\} \text { VK00037 }\end{array}$ & Drosophila melanogaster & BDSC_76607 \\
\hline y1 sc ${ }^{*}$ v1 sev21; P\{TRiP.HMS00653\}attP2 & Drosophila melanogaster & BDSC_32866 \\
\hline y1 v1; P\{TRiP.HMC02373\}attP2 & Drosophila melanogaster & BDSC_55241 \\
\hline y1 v1; P\{TRiP.HMS01939\}attP40 & Drosophila melanogaster & BDSC_39021 \\
\hline y1 v1; P\{TRiP.HMJ21356\}attP40 & Drosophila melanogaster & BDSC_53971 \\
\hline y1 v1; P\{TRiP.JF02883\}attP2 & Drosophila melanogaster & BDSC_28048 \\
\hline y1 sc ${ }^{*}$ v1 sev21; P\{TRiP.HMS01795\}attP2 & Drosophila melanogaster & BDSC_38328 \\
\hline y1 sc* v1 sev21; P\{TRiP.HMS01892\}attP40 & Drosophila melanogaster & BDSC_38976 \\
\hline y1 v1; P\{TRiP.JF01627\}attP2 & Drosophila melanogaster & BDSC_31150 \\
\hline y1 sc ${ }^{*}$ v1 sev21; P\{TRiP.HMS03722\}attP2 & Drosophila melanogaster & BDSC_54461 \\
\hline y1 v1; P\{TRiP.JF01628\}attP2 & Drosophila melanogaster & BDSC_31151 \\
\hline y1 sc ${ }^{*}$ 1 sev21; P\{TRiP.HMS02875\}attP2 & Drosophila melanogaster & BDSC_44579 \\
\hline$P\{K K 105547\}$ VIE-260B & Drosophila melanogaster & VDRC_v106127, \\
\hline$P\{K K 102631\} V I E-260 B$ & Drosophila melanogaster & VDRC_v101164, \\
\hline y1 sc* v1 sev21; P\{TRiP.HMS01858\}attP40 & Drosophila melanogaster & BDSC_38944 \\
\hline$w 1118 P\{G D 11710\} v 22180$ & Drosophila melanogaster & VDRC_v22180 \\
\hline w1118; $P\{G D 8448\} v 18396$ & Drosophila melanogaster & VDRC_V18396 \\
\hline y1 sc* v1 sev21; P\{TRiP.GL01873\}attP40 & Drosophila melanogaster & BDSC_67937 \\
\hline y1 sc ${ }^{*}$ v1 sev21; P\{TRiP.HMC06027\}attP40 & Drosophila melanogaster & BDSC_65080 \\
\hline y1 v1; P\{TRiP.HMS01824\}attP40 & Drosophila melanogaster & BDSC_38356 \\
\hline y1 sc* v1 sev21; P\{TRiP.HMS01070\}attP2 & Drosophila melanogaster & BDSC_34596 \\
\hline y1 v1; P\{TRiP.GL01278\}attP2/TM3, Sb1 & Drosophila melanogaster & BDSC_41850 \\
\hline y1 sc* v1 sev21; P\{TRiP.HMC04934\}attP40 & Drosophila melanogaster & BDSC_58190 \\
\hline y1 sc* v1 sev21; P\{TRiP.HMC04971\}attP40 & Drosophila melanogaster & BDSC_57777 \\
\hline y1 v1; P\{TRiP.HMS01821\}attP40 & Drosophila melanogaster & BDSC_38353 \\
\hline y1 sc* v1 sev21; P\{TRiP.HMC05641\}attP40 & Drosophila melanogaster & BDSC_64606 \\
\hline SJL/J & Mus musculus & $\begin{array}{l}\text { RRID:IMSR_JAX:00 } \\
0686\end{array}$ \\
\hline $\begin{array}{l}\text { B6SJL- } \\
\text { Tg(APPSwFILon, PSEN1*M146L *L286V)6799 } \\
\text { Vas/Mmjax }\end{array}$ & Mus musculus & $\begin{array}{l}\text { RRID:IMSR_JAX:03 } \\
3247\end{array}$ \\
\hline y1 w1118; PBac $\{y+-a t t P-3 B\} V K 00037$ & Drosophila melanogaster & BDSC_9752 \\
\hline \multicolumn{3}{|l|}{ Oligonucleotides } \\
\hline cindr_Exon3-4_F & CCTATGACTGGTAACCTTTTG & \\
\hline cindr_Exon4_R & GCTGCTCCAGATTCAGGTAGT & \\
\hline Ap-2a_Exon2-3_R & CCTCTTTGCTTTTGCAGTA & \\
\hline Ap-2a_Exon2_F & AGCTGAAGGGTGTGAAGTCG & \\
\hline lap_Exon8-9_F & TACGCAGTCATCAAGCTCGG & \\
\hline lap_Exon9_R & TCATAGCGGCTTCCTCTTCG & \\
\hline amph_Exon2-3_F & CATAAGATGTGTTCGCGCCG & \\
\hline amph_Exon3_R & CCACAGACTTTCCGAGGCAC & \\
\hline
\end{tabular}




\begin{tabular}{|c|c|c|}
\hline spri_Exon12-13_F & CCCTGGACTGGCGCTCCA & \\
\hline spri_Exon13_R & ACTTCACGGGTGGTGGTGT & \\
\hline Irp1_Exon5-6_R & GCTGGACAAGGACCTTTATCG & \\
\hline Irp1_Exon5_F & CAGCGGGCGCATCAATAGT & \\
\hline Ipr2_Exon9-10_F & GTAGCAAGCGTCATCTATG & \\
\hline Ipr2_Exon10_R & TGTGGCGTCCCATTCTTCTC & \\
\hline Argos_T6_attB1_F & $\begin{array}{l}\text { GGGGACAAGTTTGTACAAAAA } \\
\text { AGCAGGCTTCACCATGCCCA } \\
\text { CCACTTTAATGCTGCTGC }\end{array}$ & \\
\hline Argos_T6_attB1_R & $\begin{array}{l}\text { GGGGACCACTTTGTACAAGAA } \\
\text { AGCTGGGTCCTAGCTCTTGG } \\
\text { CGCGGGCCAGCAACTCC }\end{array}$ & \\
\hline CG34120_F & ATGGTCCTGGGTCTGATTGT & \\
\hline CG34120_R & AACACAGGCGAAGCTAAAGG & \\
\hline CG31731_F & $\begin{array}{l}\text { TTCCTAGATCCCAAGACTTAC } \\
\text { ATGA }\end{array}$ & \\
\hline CG31731_R & $\begin{array}{l}\text { GTTCAAGTTGTCCGAAATTAG } \\
\text { CG }\end{array}$ & \\
\hline ABCA_F & GTTATAGAGCTGGTGCTGCC & \\
\hline ABCA_R & GCAACGAATGTTGCAGCAGA & \\
\hline CG1494_F & AACAGGATCCACTGATGGGT & \\
\hline CG1494_R & AGATACCACTTGCCAGACGA & \\
\hline CG8908_F & AAGAAGTCGAGGACCAGTCG & \\
\hline CG8908_R & GTACTTCTCGCTGACAGTGC & \\
\hline CG31213_F & CGTGCAAACCTGAACGTACT & \\
\hline CG31213_R & CGGTTGGATAGCTTTGGCTT & \\
\hline CG42816_F & TCTGAAACCGAGTCATTGCG & \\
\hline CG42816_R & TTCCGTTTCATCCTCATGCTG & \\
\hline CG43672_F & TTACCCAAAGTCGTGATGCG & \\
\hline CG43672_R & TCCTCAACGATCAGCGTAGT & \\
\hline VPS26_Exon1-2_F & CGGTATCCGGCAAGGTGAAC & \\
\hline VPS26_Exon2_R & CGTGGTGGTTACCCCGGTCG & \\
\hline VPS35_Exon2-3_F & GCGAAGTGGCAAGACAAGTG & \\
\hline VPS35_Exon3_R & TGTCCATTCGGGTCTGCACG & \\
\hline \multicolumn{3}{|l|}{ Recombinant DNA } \\
\hline UAS-ArgosSS::Peptide & This study & \\
\hline pDONR221 entry vector & ThermoFisher & Cat \# 12536017 \\
\hline pGW-attB-HA & Bischof et al., 2012 & \\
\hline \multicolumn{3}{|l|}{ Software and Algorithms } \\
\hline FIJI & Schindelin, et al., 2012 & \\
\hline Microsoft Excel 365 & Microsoft Corporation & \\
\hline Adobe Illustrator 2020 & Adobe & \\
\hline Adobe Photoshop 2020 & Adobe & \\
\hline GraphPad Prism 8 & GraphPad & \\
\hline \multicolumn{3}{|l|}{ Other } \\
\hline A-Chamber Animal Cage Enclosure & BioSpherix & Cat \# A66274P \\
\hline ProOx 360 High Infusion Rate O2 Controller & BioSpherix & Cat \# P360 \\
\hline
\end{tabular}




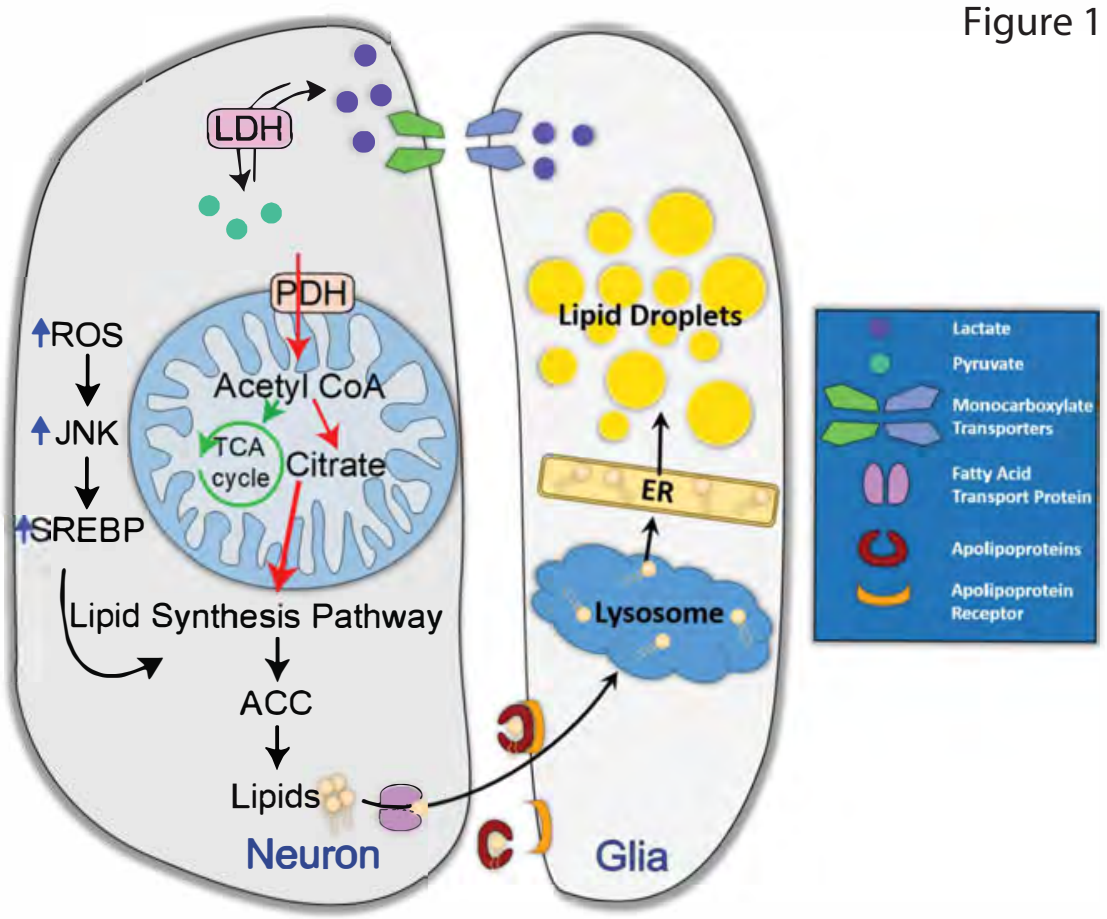




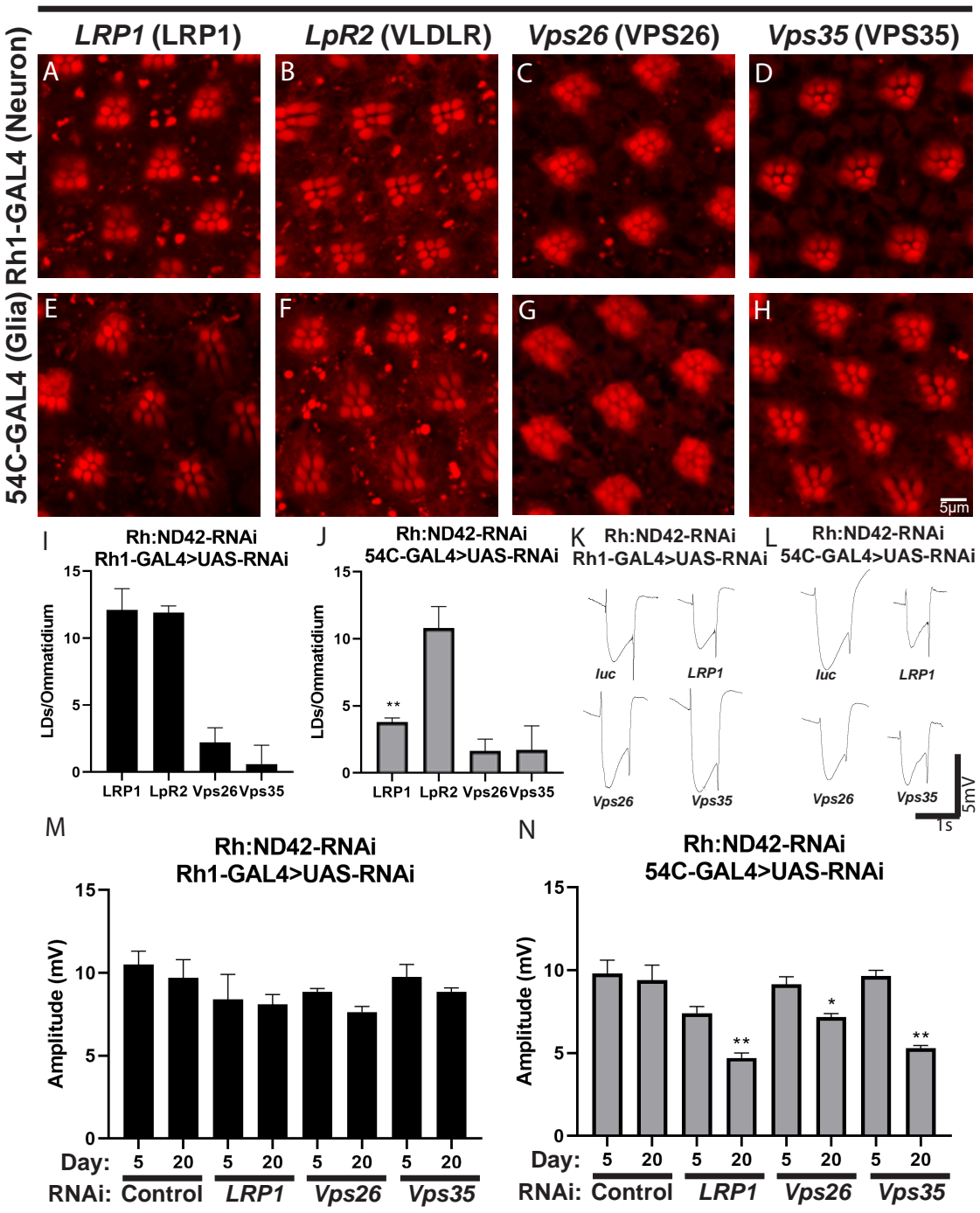


Rh-ND42 RNAi

Figure 4

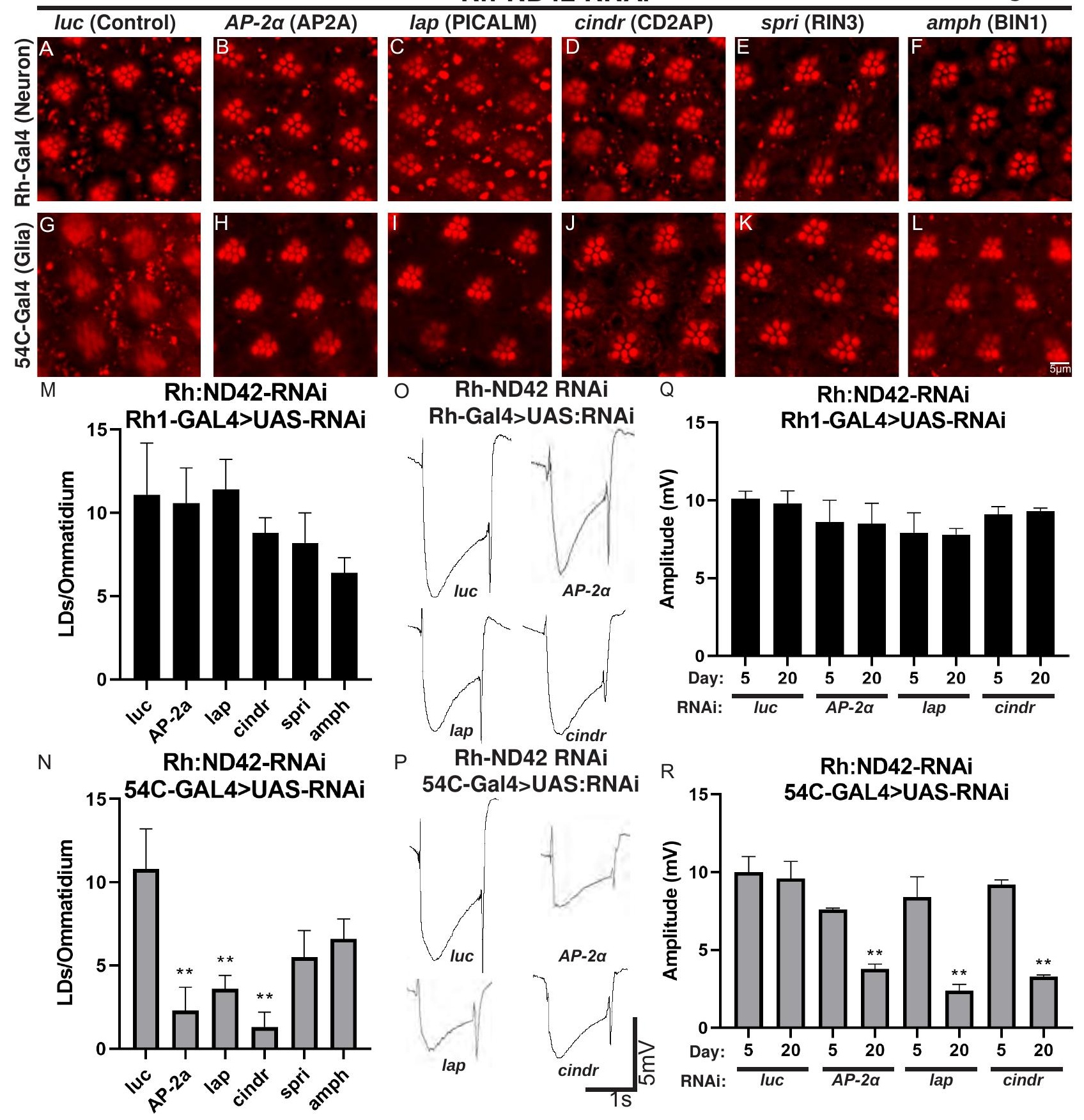


A

$\mathrm{kDa}$

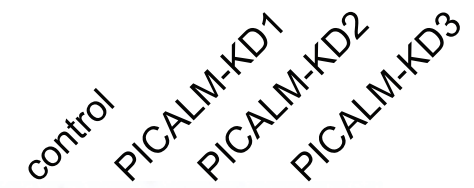

75

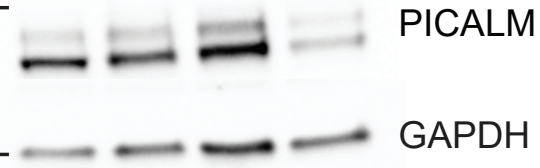

C

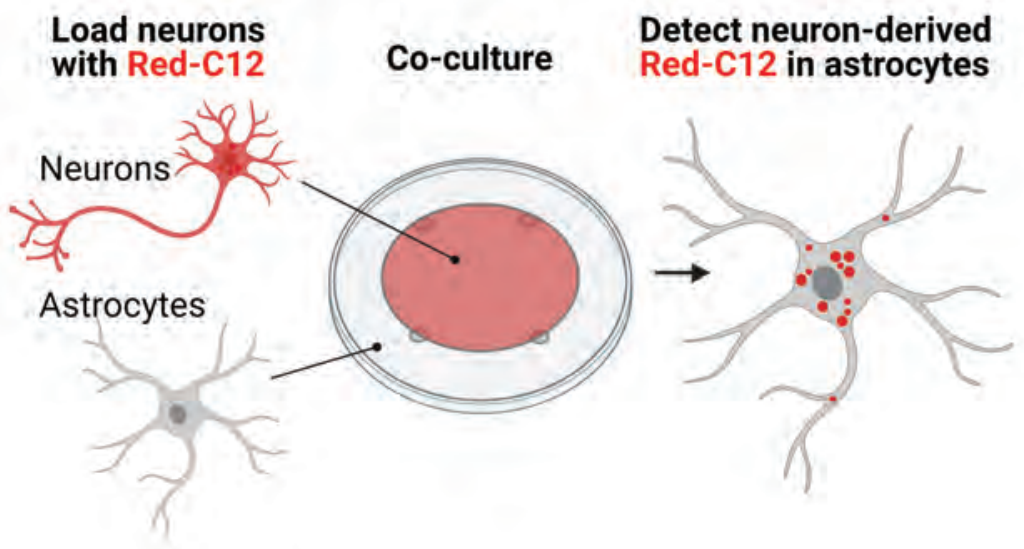

B

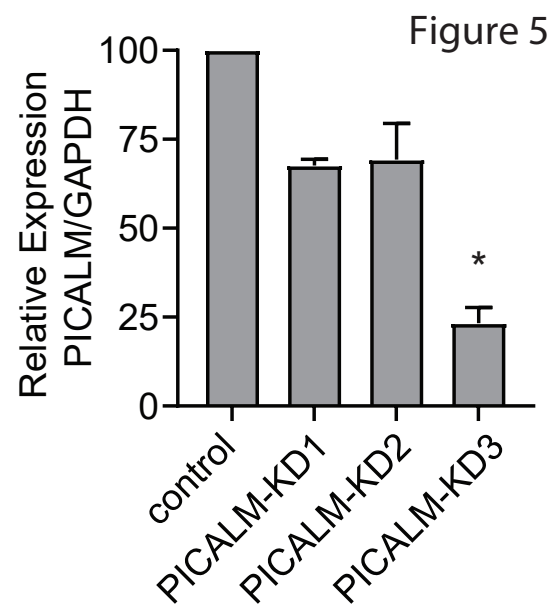

E control

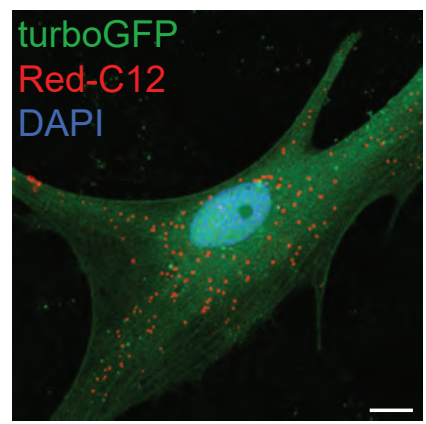

PICALM-KD1

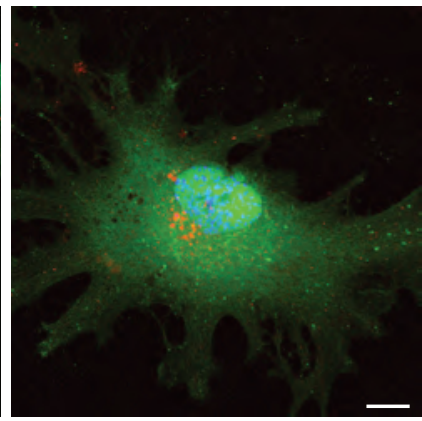

PICALM-KD2
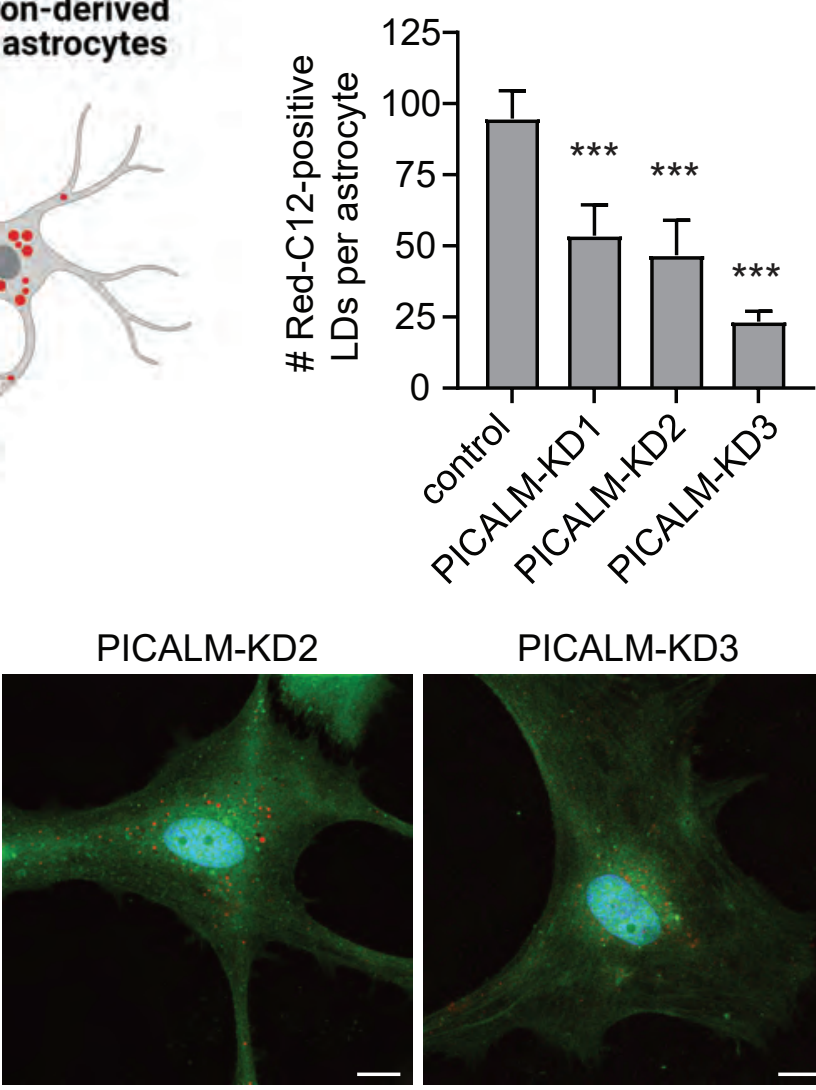

Red-C12 
Rh:marf-RNAi, Glaz-T2A-GAL4 Figure 7
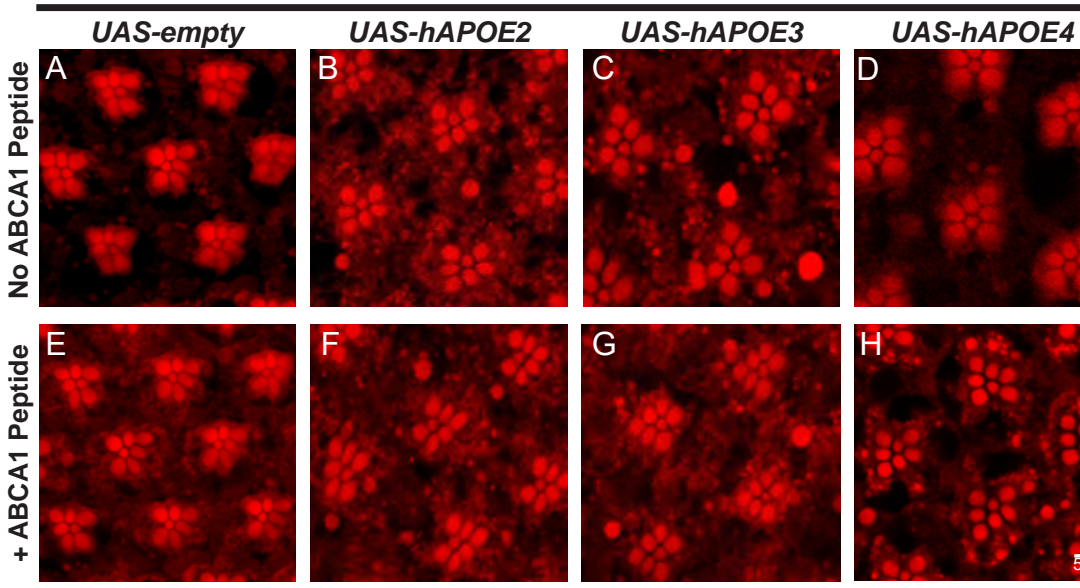

No ABCA1 Peptide
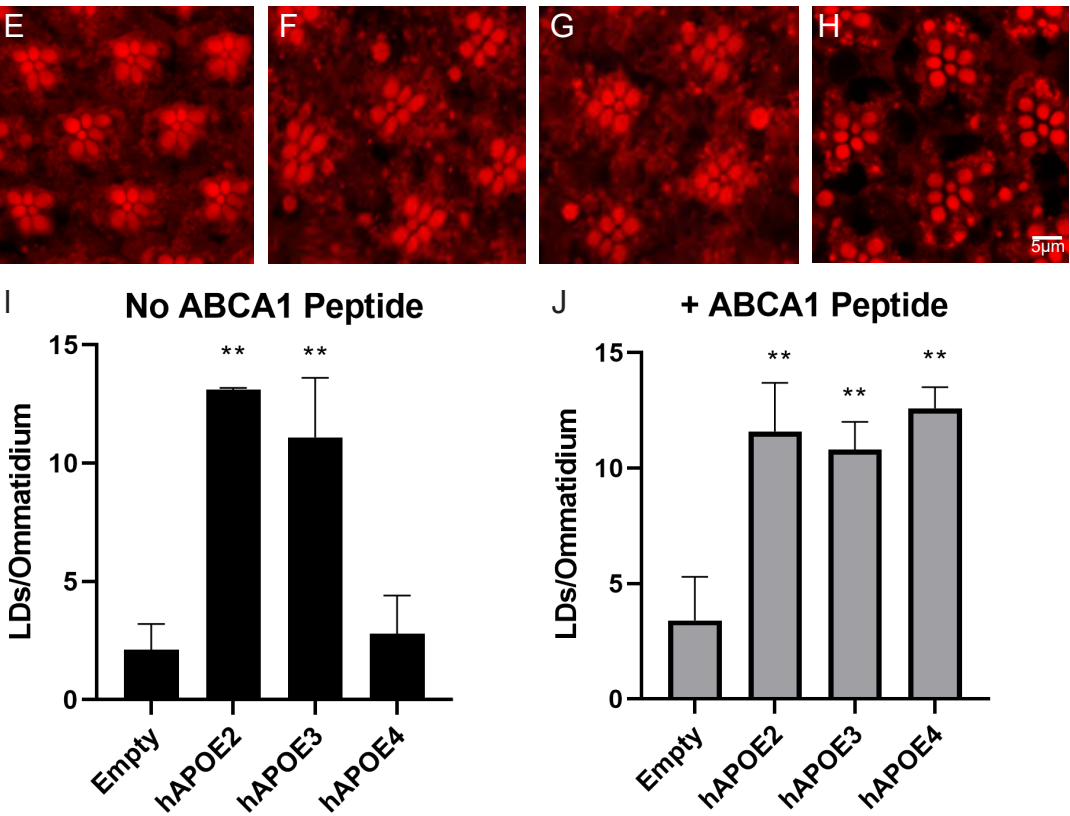

$\mathrm{J}$

+ ABCA1 Peptide

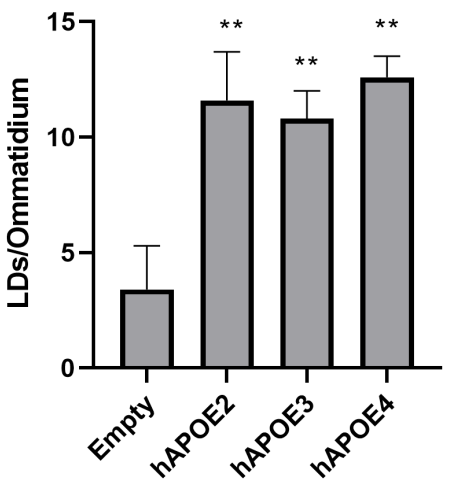




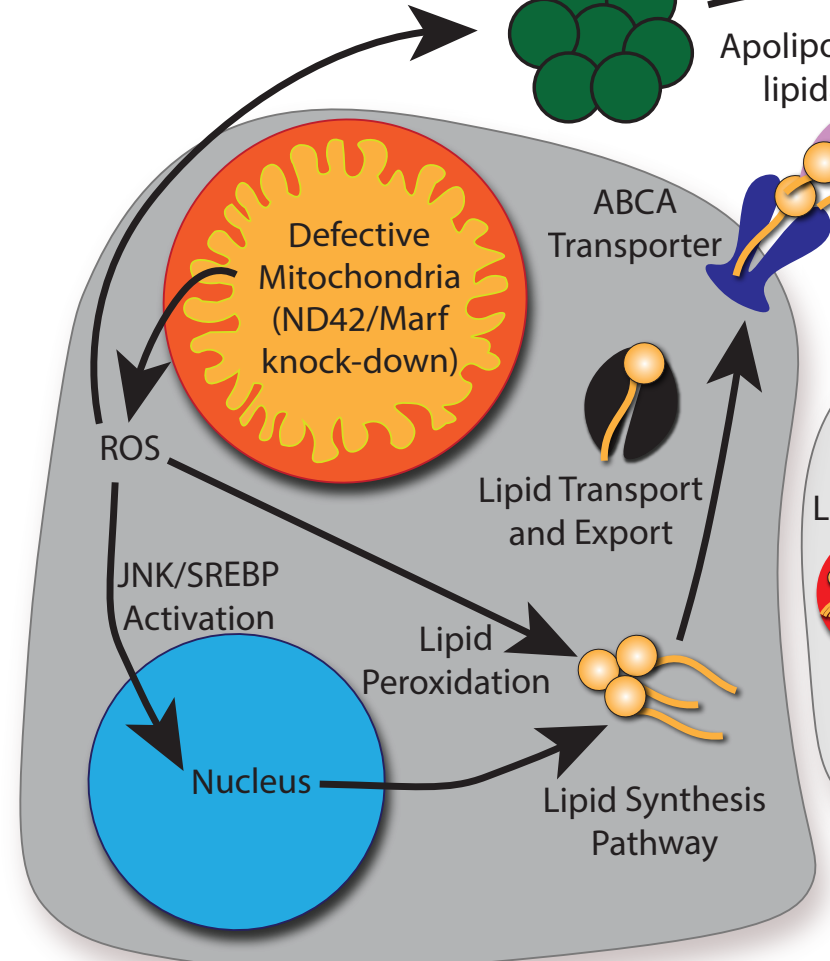

Neuron 


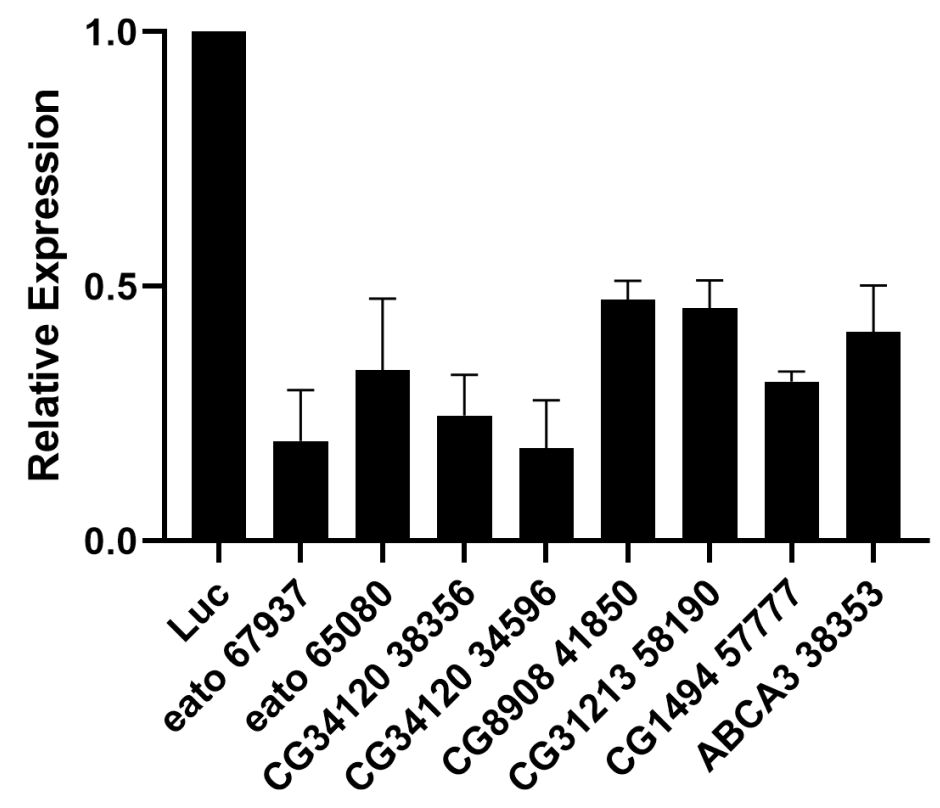

B
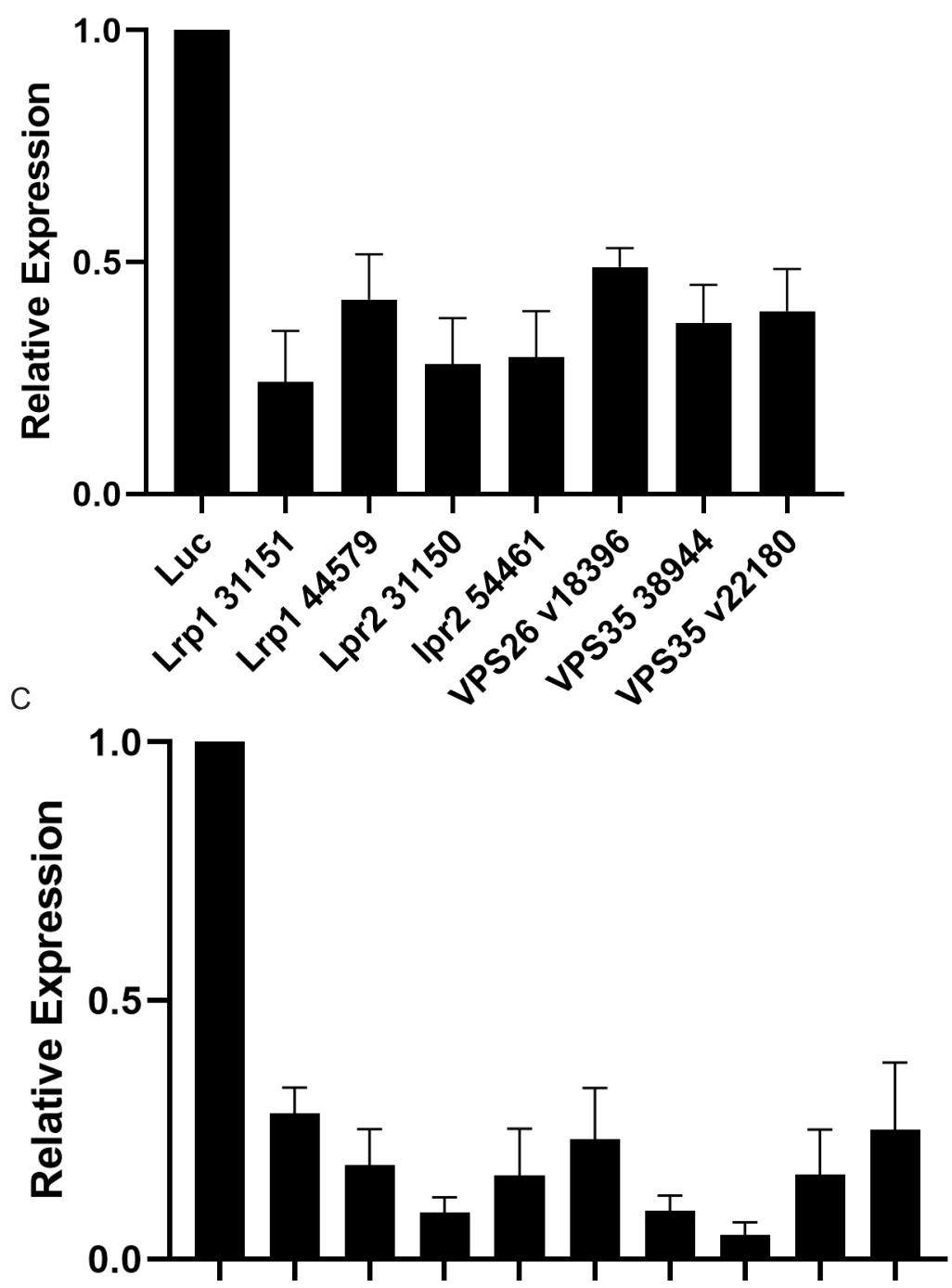

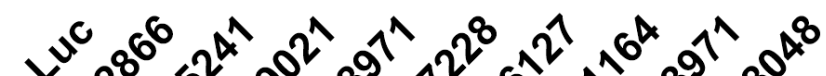

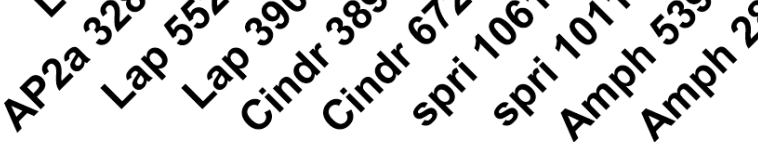


Figure Legends

2 Figure 1. Neuron-to-glia lipid transfer for lipid droplet formation.

Neurons and glia in the Drosophila visual system have a tightly regulated method of lipid production, transfer, and lipid droplet (LD) formation. Glia produce and transfer lactate through monocarboxylate transporters into neurons. Neurons convert lactate into pyruvate which feeds the TCA cycle in well-functioning mitochondrial. In defective mitochondria, ROS and citrate is produced which drives the synthesis of lipids by using Acetyl-CoA. Lipids are transported intracellularly via FatP and extracellularly via apolipoproteins where they are taken up in surrounding glia and incorporated into LDs. Adapted from Liu et al. (2017)

Figure 2. Two ABCA transporters are required in neurons for LD formation in glia.

(A) Gene tree of fly (red) and human (blue) ABCA transporter protein sequences. Human ABCA1 and ABCA7 (triangles) have been implicated as AD risk genes. Stars indicate the fly genes that were assayed in this study. Grey box indicates monophyletic grouping of closest-related fly genes to AD risk genes and their paralogs. Eato and $C G 34120$ are the closest homologs to the risk genes $A B C A 1$ and $A B C A 7$.

(B) The Drosophila eye was utilized in this study as a model of lipid transfer between neurons and glia. There are 7 visible photoreceptor neurons (with central rhabdomeres) in each optical section through a fly ommatidium, which are surrounded by pigment glia.

(C-J) Lipid droplet analysis in fly retina. To induce ROS specifically in photoreceptor neurons, an RNAi against ND42, a mitochondrial complex I subunit is expressed under the control of the Rhodopsin (Rh)-GAL4 driver. Animals are reared at $29^{\circ} \mathrm{C}$ under $12-$ hour light/dark conditions. ROS in neurons induces glial LD formation in control animals $(\mathrm{C}$ and $\mathrm{F})$. The photoreceptor rhabdomeres stain positive with Nile red but photoreceptors (dashed lines) do not accumulate LD. In contrast, pigment glia accumulate LD (arrowheads). Knockdown of Eato and CG34120 in neurons (D-E), but not in glia (G-H), suppress LD formation, quantified in (I-J), demonstrating a critical role for these genes in neurons for LD formation.

(K-N) To assess the functional consequences of the lack of LD formation we performed electroretinograms (ERGs) at day 5 and day 20. Animals were housed at $29^{\circ} \mathrm{C}$ under $12-$ hour light/dark conditions, $\mathrm{n} \geq 10$ animals per genotype. Representative ERG traces from animals with genotypes indicated. ERG amplitude quantification (K and L) show that glial knock down of either Eato or CG34120 gene tested does not affect ERG amplitude. However, neuronal knock-down of these genes lead to a dramatic reduction of ERG amplitude over time, showing a progressive neurodegeneration. Hence, ROS induced LD formation in glia provide a protection against neurodegeneration.

Figure 3. The APOE receptor, LRP1, and retromer components Vps 26 and Vps35 are required for LD formation.

(A-J) LD analysis in fly retina. ROS is induced in neurons and RNAi directed against the apolipoprotein receptors ( $L R P 1$ and $L p R 2$ ) or genes critical for retromer function (Vps26 and $V p s 35)$ are expressed in neurons (Rh-Gal4, A-D) and pigment glia (54c-Gal4, E-H). Animals were reared at $29^{\circ} \mathrm{C}$ under 12 -hour light/dark conditions. LRP1 is required in glia (E) but not in neurons (A) to form LD whereas LpR2 is not required in either cell (B, 
F). In contrast, the retromer proteins are required in both neurons and glia to form LD (C$\mathrm{D}, \mathrm{G}-\mathrm{H})$. LD number per ommatidium is quantified (I-J).

Figure 4. Alzheimer's disease-associated GWAS genes are required in glia for LD formation upon neuronal ROS induction.

(A-N) Lipid droplet analysis in fly retina. ROS is induced in neurons and RNAi directed against homologs of 5 GWAS genes in photoreceptor neurons (A-F) or glia (G-L). Animals are housed at $29^{\circ} \mathrm{C}$ under 12-hour light/dark conditions. Expression of RNAi against any genes tested in neurons do not affect the formation of LD in glia significantly (A-F). In contrast, RNAi targeting $A P-2 a$, lap, and cindr (but not in a statistically significant manner in spri and $a m p h$ ) in glia reduced LD formation significantly (G-L) as quantified (M-N).

(O-R) ERG assays were performed to assess neurodegeneration. Animals are housed at $29^{\circ} \mathrm{C}$ under 12-hour light/dark conditions, $n \geq 10$ animals per genotype. Representative traces (O-P) and amplitude quantification (Q-R) demonstrate that neuronal knock down of any gene tested does not affect ERG amplitude. In contrast, glial knock-down of the genes lead to a reduction in LD formation ( $A P-2 a$, lap, and cindr) led to a significant reduction of ERG amplitude over time, showing a progressive neurodegeneration. Hence, these genes are required in glia to take up peroxidated lipids and their loss promotes neurodegeneration.

Figure 5. Lipid transfer between neurons and astrocytes is blunted by knockdown of PICALM.

(A) Astrocytes were transduced with lentivirus expressing non-targeting shRNA (control), or three independent PICALM targeting shRNAs (KD1-3). Cell lysates were analyzed by Western blot for PICALM levels and GAPDH as a loading control.

(B) Levels of PICALM from transduced astrocytes were quantified and normalized to GAPDH control. Mean \pm SEM, Kruskal-Wallis test with Dunn's posttest * $p=0.05$ compared to control, $\mathrm{n}=3$ from three independent experiments.

(C) Schematic of Red-C12 transfer assay.

(D) Quantification of Red-C12-positive lipid droplets (LDs) in astrocytes. Mean $\pm \mathrm{SEM}$, One-way ANOVA with Dunnett's posttest $* * * \mathrm{p}<0.001$ compared to control, $\mathrm{n}=6$ from three independent experiments.

(E) Representative maximum intensity projections of confocal images of transduced astrocytes following the assay. TurboGFP reporter expression marks transduced cells. Scale bars are $10 \mu \mathrm{m}$.

Figure 6. Elevated ROS and the presence of A 342 synergize to induce neurodegeneration in flies 90 and mice. 
(A-F) Lipid droplet analysis in fly retina. Animals are housed at $29^{\circ} \mathrm{C}$ under 12 -hour light/dark conditions with food changed daily; representative images of $\geq 10$ animals per genotype. Wild-type flies exposed to $25 \mu \mathrm{M}$ rotenone food at (A) 1 day or (D) 10 days post eclosion were compared with A $\beta 42$-expressing flies at (B) 1 day post eclosion or (E) 10 days post eclosion and with $A \beta 42$-expressing flies exposed to $25 \mu \mathrm{M}$ rotenone food at (C) 1 day post eclosion and (F) 10 days post eclosion. Note the absence of LD formation with either treatment but the dramatic increase in diffuse Nile red staining and the demise of PR by day 10 showing that ROS and A $\beta 42$ synergize to cause the demise of neurons.

(G-L) A 342 immunohistochemical analysis of 4 mo. old mouse brain sections from wildtype mice reared in normoxic $(\mathrm{G})$ or hyperoxic $(\mathrm{H})$ conditions compared to 5XFAD mice reared in normoxic (I) or hyperoxic $(\mathrm{J})$ conditions for 3 mos. prior to sacrifice. Arrowheads indicate plaques, $n=3 /$ genotype and treatment condition. Quantification of average plaque number $(\mathrm{K})$ or plaque size $(\mathrm{L})$ in the subiculum of mice from G-J is elevated in $A \beta$-expressing mice exposed to hyperoxia, showing that ROS induction enhances plaque formation.

Figure 7. An ABCA1 agonist peptide rescues LD formation in the presence of APOE4.

(A-J) LD analysis in fly retina. ROS was induced in photoreceptor neurons, as previously reported (Liu et al., 2015, 2017), using an RNAi against marf, the fly ortholog of mitofusin, under the control of Rh-GAL4. Animals are reared at $29^{\circ} \mathrm{C}$ under 12 -hour light/dark conditions; representative images of $\geq 10$ animals per genotype. We utilized a previously characterized allele of Glial Lazarillo (Glaz-T2A:Gal4). LD formation is inhibited in Glaz-T2A-Gal4/+ flies but can be restored by expressing human APOE2 or APOE3, but not APOE4. An ABCA1 agonist peptide was genetically encoded in the fly and expressed in the human APOE variant flies to assess LD formation. Expression of the peptide does not affect LD formation in the presence of APOE2 or APOE3, but fully restores LD formation in the APOE4 expressing flies (E-H) and quantified (I-J) showing that $\mathrm{LD}$ formation is strongly enhanced by this peptide.

Figure 8. Model of lipid droplet accumulation and players identified in this study. We propose a model in which genetic (loss of ABCA, endocytic, or retromer genes) together with environmental insults (ROS) sensitize neurons to the presence of Amyloid accumulation to induce neurodegeneration. It is likely that this synergy between multiple insults severely exacerbates neuronal loss in disease. We demonstrated that lipid transfer between neurons and glia requires neuronal ABCA transporters, glial apolipoprotein receptors, and the retromer, which is required for LRP1 recycling. We propose that endocytosis of lipid particles are processed through lysosomes upon endocytosis. Lysosomes degrade $A \beta 42$ and the lipids are shuttled to the ER to form LD. Hence, this transport of peroxidated lipids and $A \beta 42$ provide dual protective effects.

Supplemental Figure 1. Analysis of RNA expression of genes targeted in this study. (A-C) Quantitation of mRNA expression of the genes assayed after RNAi induction in fly heads. Animals are housed at $29^{\circ} \mathrm{C}$ under 12 -hour light/dark conditions. In each assay, a luciferase 
bioRxiv preprint doi: https://doi.org/10.1101/2021.03.03.433580; this version posted March 4, 2021. The copyright holder for this preprint (which was not certified by peer review) is the author/funder, who has granted bioRxiv a license to display the preprint in perpetuity. It is made available under aCC-BY-NC-ND 4.0 International license.

$135 \mathrm{RNAi}$ is used as a negative control. RNAi induces at least a 50\% reduction of the mRNA of all 136 genes assayed. 